\title{
A competitive strategy for atrial and aortic tract segmentation based on deformable models
}

\author{
Pedro Morais ${ }^{\mathrm{a}, \mathrm{b}, c, *}$, João L. Vilaça ${ }^{\mathrm{b}, \mathrm{d}}$, Sandro Queirós ${ }^{\mathrm{b}, c, e}$, Felix Bourier ${ }^{\mathrm{f}}$, Isabel Deisenhofer ${ }^{\mathrm{f}}$, \\ João Manuel R.S. Tavares ${ }^{a}$, Jan D'hooge ${ }^{c}$ \\ a Instituto de Ciência e Inovação em Engenharia Mecânica e Engenharia Industrial, Faculdade de Engenharia, Universidade do Porto, Porto, Portugal \\ ' ICVS/3B's - PT Government Associate Laboratory, Braga/Guimarães, Portugal \\ ${ }^{c}$ Lab on Cardiovascular Imaging \& Dynamics, Department of Cardiovascular Sciences, KULeuven - University of Leuven, Leuven, Belgium \\ ${ }^{\mathrm{d}}$ DIGARC - Polytechnic Institute of Cávado and Ave, Barcelos, Portugal \\ e Algoritmi Center, School of Engineering, University of Minho, Guimarães, Portugal \\ ${ }^{\mathrm{f}}$ Department of Electrophysiology, German Heart Center Munich, Technical University, Munich, Germany
}

\section{A R T I C L E I N F O}

\section{Article history:}

Received 31 January 2017

Revised 30 June 2017

Accepted 26 July 2017

Available online 27 July 2017

\section{Keywords:}

Image segmentation

Competitive contours

Atrial and aortic tract segmentation

B-spline explicit active surfaces

\begin{abstract}
A B S T R A C T
Multiple strategies have previously been described for atrial region (i.e. atrial bodies and aortic tract) segmentation. Although these techniques have proven their accuracy, inadequate results in the mid atrial walls are common, restricting their application for specific cardiac interventions. In this work, we introduce a novel competitive strategy to perform atrial region segmentation with correct delineation of the thin mid walls, and integrated it into the B-spline Explicit Active Surfaces framework. A doublestage segmentation process is used, which starts with a fast contour growing followed by a refinement stage with local descriptors. Independent functions are used to define each region, being afterward combined to compete for the optimal boundary. The competition locally constrains the surface evolution, prevents overlaps and allows refinement to the walls. Three different scenarios were used to demonstrate the advantages of the proposed approach, through the evaluation of its segmentation accuracy, and its performance for heterogeneous mid walls. Both computed tomography and magnetic resonance imaging datasets were used, presenting results similar to the state-of-the-art methods for both atria and aorta. The competitive strategy showed its superior performance with statistically significant differences against the traditional free-evolution approach in cases with bad image quality or missed atrial/aortic walls. Moreover, only the competitive approach was able to accurately segment the atrial/aortic wall. Overall, the proposed strategy showed to be suitable for atrial region segmentation with a correct segmentation of the mid thin walls, demonstrating its added value with respect to the traditional techniques.
\end{abstract}

(c) 2017 Elsevier B.V. All rights reserved.

\section{Introduction}

Anatomical assessment of the atrial region (i.e. atrial bodies and aortic tract - Ao) through medical imaging has gained particular interest during the last decade. Several authors extracted/isolated multiple contours of the atrial anatomies using different segmentation strategies (Tobon-Gomez et al., 2015), proving the clinical relevance of a correct anatomical and functional assessment of each atrial region for global cardiac function quantification and even for risk stratification (Hoit, 2014; Melenovsky et al., 2014). Moreover, enhanced minimally invasive cardiac interventions were proposed, using electroanatomic mapping techniques (Rolf et al.,

\footnotetext{
* Corresponding author at: Life and Health Sciences Research Institute (ICVS), Universidade do Minho, Campus de Gualtar, 4710-057 Braga, Portugal.

E-mail address: pedromorais@med.uminho.pt (P. Morais).
}

2014) or even superimposing pre-operative anatomical atrial surfaces (Bourier et al., 2016), extracted from highly detailed datasets, into intra-operative imaging (e.g. fluoroscopy). In fact, the fusion of pre- and intra-operative data is an emergent research topic, where the limitations commonly associated with the intra-operative data (e.g. difficult to detect the cardiac boundaries, or the small field-ofview) are reduced, facilitating the entire procedure and making it safer even in inexperienced hands.

Automatic and semi-automatic atrial region segmentation solutions have been explored and presented for multiple imaging modalities, such as computed tomography (CT, (Ecabert et al., 2011; Kirișli et al., 2010; Zheng et al., 2008)) and magnetic resonance imaging (MRI, (Zuluaga et al., 2013)). Since the manual approach is tedious, time-consuming and has a high intra- and inter-observer variability, automated segmentation processes have been widely explored and increasingly accepted in normal clinical practice. The 
majority of the applied methods are based on deformable models (Ecabert et al., 2011), atlas-based techniques (Kirişli et al., 2010; Zuluaga et al., 2013) and machine learning (Zheng et al., 2008), proving its high accuracy in a high number of cases with different pathologies. Some studies focused only on the left atrium (LA) due to its importance for atrial fibrillation, using a simple initialization strategy through a multi-atlas to obtain a rough contour alignment (Sandoval et al., 2013) or a probabilistic atlas (Stender et al., 2013), followed by a region growing and multiple 2D individual segmentations with circular shape descriptors (Ammar et al., 2013). Moreover, Zuluaga et al. (2013) presented an atlas-based technique with global and deformable alignment for LA segmentation only. Nevertheless, the current solutions show inaccurate results in thin septal walls (Zhuang et al., 2010) or present a total merge/overlap between atrial contours (Kirişli et al., 2010; Zuluaga et al., 2013). Specifically, the atlas-based technique with a final majority voting per chamber approach presented by Kirişli et al. (2010) was unable to prevent overlap between contours. Contrarily, the atlas-based approach from Zuluaga et al. (2013) and the deformable model proposed by (Ecabert et al., 2011) prevented overlapping regions by simply merging these regions. Thus, accurate assessment of the thin walls is currently not possible, missing their use for specific diagnostic purposes and interventional planning related with the thin atrial walls, e.g. transseptal puncture (Morais et al., 2017).

Multiple strategies have been proposed to segment multiple structures in different scenarios, using different formulations to identify the target regions and prevent overlap and gap regions between contours (Jimenez-del-Toro et al., 2016). Initially, several authors extended their individual structure methods to multistructures. Some examples are the atlas-based (Okada et al., 2015; Wolz et al., 2013; Xu et al., 2015), and statistical-based approaches (Yan et al., 2005; Yang et al., 2004). Despite the high versatility obtained, overlapping and merged regions were typically found, requiring post-processing techniques through mathematical morphology operations or refinement methodologies (Iglesias and Sabuncu, 2015).

Cooperative strategies (e.g., coupled level sets) were also presented and showed their robustness for multiple structure situations (Yezzi et al., 2002), such as endo- and epicardial left ventricular wall segmentation (Alessandrini et al., 2009; Pedrosa et al., 2017; Queirós et al., 2014). These strategies use multiple functions (one per target region) and combine them during the optimization. Each individual curve is affected by the remaining ones, consequently cooperating to maximize/minimize the functional energy. As such, these strategies are less sensitive to local minima and to the initialization when compared with multiple independent segmentation approaches (Chen et al., 2008; Yezzi et al., 2002). However, these models do not have an intrinsic restriction to prevent overlapping regions (Faisal et al., 2015), consequently requiring several and complex penalty terms (Barbosa et al., 2010) to obtain mutual exclusiveness and boundary share between the different contours (Faisal et al., 2015).

On the contrary, competitive contours use a different multistructure segmentation paradigm, where the multiple curves that define the target structures interact between them at the contour boundaries to avoid overlapping regions (Brox and Weickert, 2006; Lankton and Tannenbaum, 2008). This interaction is usually performed through act-react strategies (Brox and Weickert, 2006; Lankton and Tannenbaum, 2008), where the contour with stronger force controls the update of the remaining ones. Nevertheless, at regions of no competition, external terms (e.g., constant growing (Brox and Weickert, 2006)) are required to prevent empty regions between contours. Initially, these methods were applied for the entire image domain, failing to segment small regions of the image or at least requiring an extra function that represents the back- ground (Gao et al., 2012). As such, these strategies were suboptimal for medical imaging problems, where only a few number of organs are typically assessed per study. In this sense, Gao et al. (2012) presented a local robust statistic driven active contour that uses act-react forces to perform interactive segmentation in small portions of the image. Since full union between contours was not required, this solution proved its added value for the segmentation of regions with thin walls. Nevertheless, this generic framework presented some limitations to perform accurate segmentation of thin walls with low contrast, generating small bridges/connections between contours.

In this work, we present a novel competitive contours approach for segmentation of multiple structures. The novel method is applied for atrial region segmentation with correct delineation of the thin mid atrial walls, even in low contrast scenarios. Multiple independent functions are used to control each contour (i.e. LA, Ao and right atrium - RA), being afterwards combined to compete for the optimal boundary transition. Indeed, the competition is only applied when two or more contours are near each other, penalizing the surface evolution based on the local distance between contours. As such, fast contour growing is prevented, and a refinement to the thin wall is achieved. Although this competitive methodology is generic and suitable to be applied in several frameworks, we decided to prove its advantages using the B-spline Explicit Active Surfaces (BEAS) framework (Barbosa et al., 2012), which proved to be suitable for individual cardiac chamber segmentation, such as the left ventricle (Queirós et al., 2014) and the aorta (Queirós et al., 2017; Queirós et al., 2016). It should be noticed that, although coupled BEAS strategies have been proposed for myocardial segmentation using concentric contours (Pedrosa et al., 2017; Queirós et al., 2014), generic competitive approaches that allow segmentation of multiple structures without shape/model restrictions are still missing.

Hereupon, the current work introduces three novelties, namely: 1) a new competitive strategy that allows accurate segmentation of multi-structures with thin and heterogeneous mid walls; 2) a novel methodology to segment the atrial region in multiple imaging modalities based on the proposed competitive strategy; and 3) exhaustive validation of the novel competitive method for atrial region segmentation.

This paper is structured as follows. In Section 2, a technical description of the proposed strategy for atrial region segmentation is presented, followed by an explanation of the proposed competitive technique. In Section 3, the validation experiments and their results are presented. Section 4 evaluates and discusses the performance of the proposed competitive method against state-of-the-art techniques and free-evolution approaches. Finally, the conclusions of the work are presented in Section 5.

\section{Methods}

In this section, we present the strategy used to segment the LA, RA and Ao. The proposed semi-automatic method relies on three consecutive stages (Fig. 1): 1) manual identification of the different regions through one click in each chamber and three clicks in the aortic tract; 2) fast growing of the contours using a BEASthreshold strategy (Section 2.1.4); and 3) contour refinement using BEAS-segmentation (Section 2.1.5) with localized energies.

The clicked positions are used to initialize two ideal spheres (for LA and RA) and one cylinder (for Ao), which are afterwards adapted to the anatomy. Each individual click defines the center position of a sphere with a radius of approximately $15 \mathrm{~mm}$ and the three clicks in the Ao define the centerline of a cylinder with a radius of approximately $5 \mathrm{~mm}$. Furthermore, and in order to identify the mitral and tricuspid valve region, both left and right ventricles (LV and RV) are also initialized (two ideal spheres with radius of 


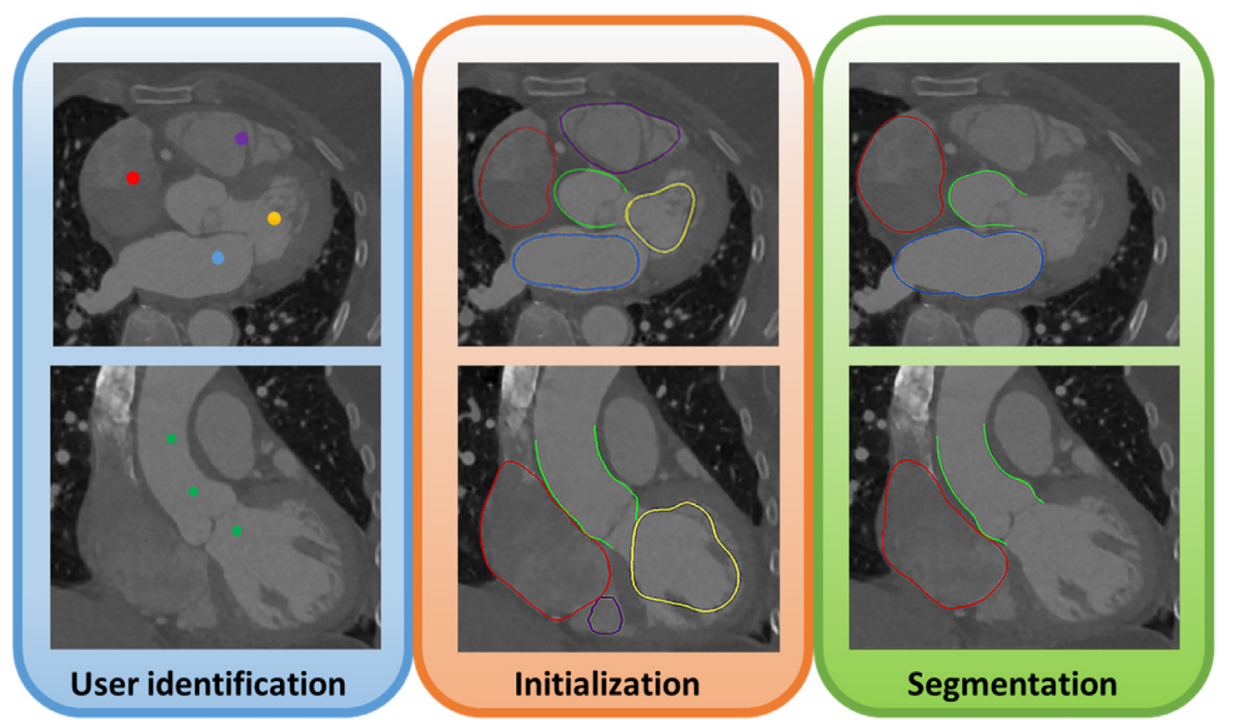

Fig. 1. Overview of the proposed competitive strategy for atrial region segmentation.

$5 \mathrm{~mm}$ ) with one click in each blood pool. The spatial location of both ventricles is used to locally constrain the atrial surfaces, preventing their evolution inside the ventricle regions (further details in Section 2.2.2).

The BEAS-threshold is first used to grow the initialized contour (i.e. the cylinder and spheres) using global descriptors, minimizing the influence of the initialization on the segmentation result. The segmentation is then applied to refine the current surfaces and to accurately delineate the atrial and aortic walls. In order to increase the robustness of the technique, edge-based (Section 2.1.2) and simple shape regularization terms (Section 2.1.3) are used during the entire process. The edge-based terms reduce the influence of the initialization and guide the segmentation in heterogeneous and vacuum regions, while the regularization term uses the curvature information of each contour to regularize the contour and to reduce the degrees of freedom of the segmentation. Moreover, the regularization term controls the contour evolution in vacuum regions (e.g., tricuspid valve).

Two independent implementations are presented, one where each structure evolves independently (henceforward mentioned as free-evolution BEAS, Section 2.1), and a second where a novel competitive approach is presented, allowing interaction between the contours while guaranteeing the integrity of the thin mid atrial walls (competitive BEAS, Section 2.2).

It should be noticed that both ventricles (LV and RA) are only evolved in the threshold-based stage (Section 2.1.4, Fig. 1), allowing their local competition with the atrial chambers and consequently defining the valve plane.

\subsection{Free evolution B-spline explicit active surfaces}

\subsubsection{B-spline explicit active surfaces}

The BEAS framework was initially proposed in Barbosa et al. (2012), having as key novelty the representation of the interface as an explicit function described using B-spline coefficients $(c[\boldsymbol{k}])$. This implies that one of the coordinates of the points of the interface, $\boldsymbol{x}=\left\{x_{1}, x_{2}, \ldots, x_{N}\right\}$ in a $N$-dimensional space, is expressed as a function of the remaining coordinates (i.e. $\boldsymbol{x}^{*}=\left\{x_{2}, \ldots, x_{N}\right\}$ ) consequently reducing the dimensionality of the segmentation problem and intrinsically including shape limitations with clear advantages for non-complex shapes.
The explicit function $\psi$ is therefore defined as (Almeida et al., 2016):

$x_{1}=\psi\left(\boldsymbol{x}^{*}\right)=\sum_{k \in \mathbb{Z}^{N-1}} c[\boldsymbol{k}] \beta^{d}\left(\frac{\boldsymbol{x}^{*}-\boldsymbol{k} h_{r}}{h_{s}}\right)$,

where $\beta^{d}(\cdot)$ is the uniform symmetric $(N-1)$-dimensional Bspline of degree $d$. The knots of the B-splines are located on a regular grid defined on a specific space (e.g., polar or cylindrical space). $h_{s}$ and $h_{r}$ are smoothness parameters that control the scaling and spacing of the B-spline kernel, respectively. Specifically for atrial region segmentation, both atria are described through a spherical model, while the aorta tract is represented by a cylindrical one.

Regarding the contour evolution, multiple energies are used to control the optimization process of each contour $i$. Two strategies can be used to optimize the multiple energy terms. The first approach minimizes each energy individually, therefore allowing that one energy converges before the second one. Contrarily, the second approach combines both terms and the iterative process is only stopped when both contours converge. Due to the simplicity of the method (mainly when more than two contours are used), we decided to prove the advantage of our competitive technique using the second strategy. Nonetheless, note that the independent optimization approach could also be implemented. In this sense, the BEAS energy $(E)$ for multiple contours is computed through:

$E=\sum_{i} E_{i}$

$E_{i}=\int_{\Omega} \delta_{\phi_{i}}(\boldsymbol{x}) \int_{\Omega} B(\boldsymbol{x}, \boldsymbol{y}) \cdot F_{i}(\boldsymbol{y}) d \boldsymbol{y} d \boldsymbol{x}$

where $\phi_{i}(\boldsymbol{x})=\Gamma_{i}\left(\boldsymbol{x}^{*}\right)-x_{1}, i \in\{1, \ldots, n\}$ with $n$ representing the total number of contours, $\boldsymbol{x}, \boldsymbol{y}$ are independent spatial locations in the image domain $\Omega, \phi_{i}(\boldsymbol{x})$ is a level-set like function representing the region inside the interface $\Gamma_{i}, \delta_{\phi_{i}}$ is the dirac function and $F_{i}$ an image criteria (e.g. localized Chan-Vese energy, (Lankton and Tannenbaum, 2008)). $B(\boldsymbol{x}, \boldsymbol{y})$ corresponds to a mask function in which the regional parameters that locally drive the contour evolutions are computed and it is defined as the set of points belonging to the normal direction of $\boldsymbol{x}$ and whose distance is lower than $\rho$. Furthermore, the energy is minimized through the computation of the energy derivatives with respect to each B-spline coefficient for each contour $i$, through (Barbosa et al., 2012):

$$
\frac{\partial E_{i}}{\partial c_{i}[\boldsymbol{k}]}=\int_{\Gamma_{i}} \bar{g}_{i}\left(\boldsymbol{x}^{*}\right) \beta_{i}^{d}\left(\frac{\boldsymbol{x}^{*}-\boldsymbol{k} h_{r i}}{h_{s i}}\right) d \boldsymbol{x}^{*},
$$



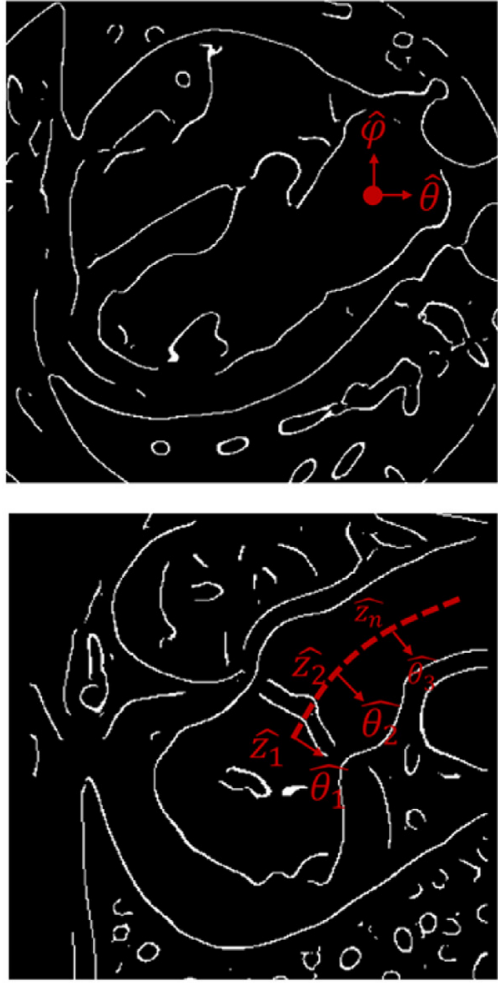

(a)
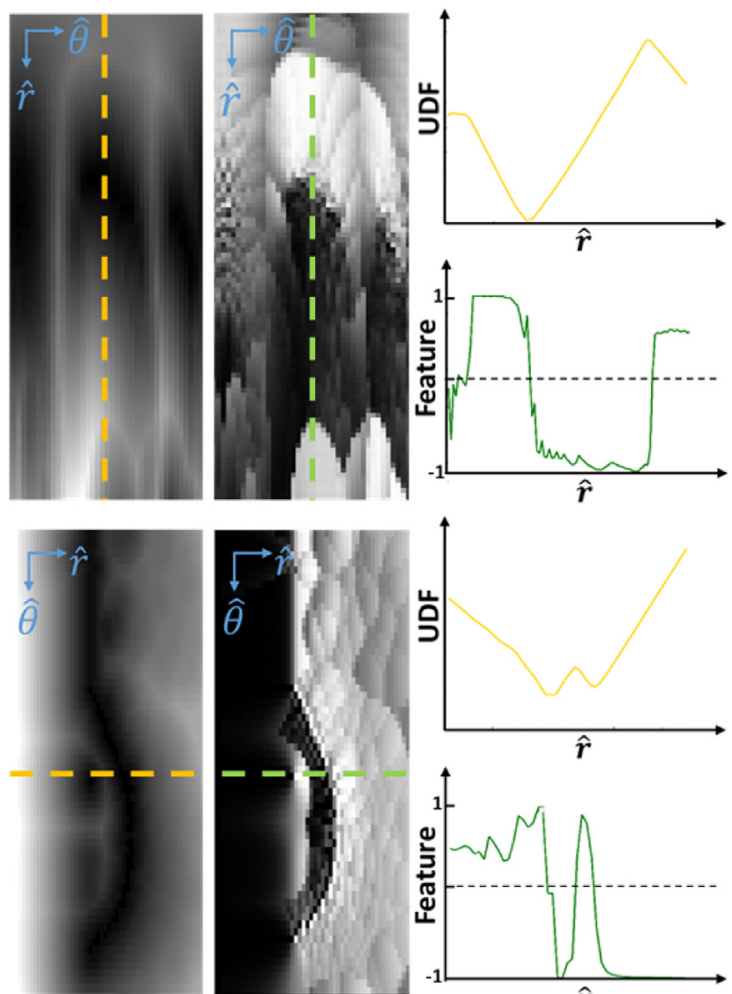

(b)

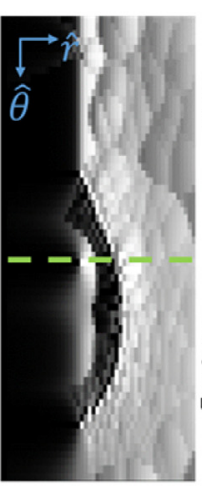

(c)
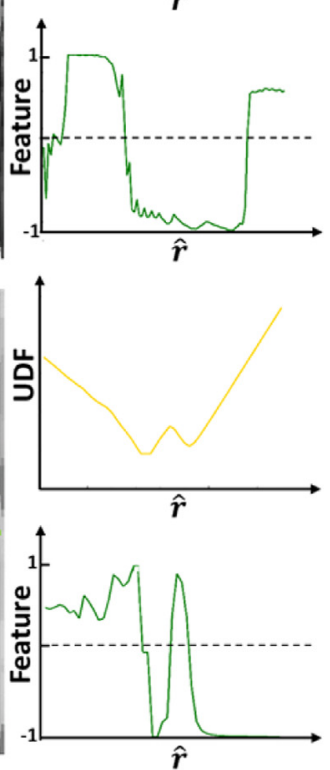

(d)

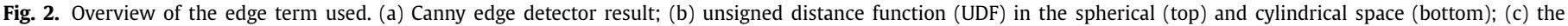
respective feature; (d) profile of the energy (UDF) and the feature used during the segmentation.

with $\bar{g}_{i}\left(\boldsymbol{x}^{*}\right)$ representing the feature map (e.g. first derivative of the local Chan-Vese energy, (Lankton and Tannenbaum, 2008)) of each contour $i$. The traditional formulation from BEAS used regional intensity-based terms directly extracted from the image $\left(\bar{g}^{I M}\right)$, mathematically described as image criteria $F^{R}$ and feature $\overline{g^{R}}$. As such, Eqs. (2) and (3) are updated to:

$F_{i}(\boldsymbol{y})=F^{R}(\boldsymbol{y})$,

$\bar{g}_{i}\left(\boldsymbol{x}^{*}\right)=\bar{g}_{i}^{I M}\left(\boldsymbol{x}^{*}\right)=\overline{g^{R}}\left(\boldsymbol{x}^{*}\right)$,

The reader is kindly directed to the Barbosa et al. (2012) for further details on BEAS.

\subsubsection{Edge based energy term}

Queirós et al. (2014) presented and integrated an edge-based term in BEAS to segment $2 \mathrm{D}+\mathrm{t}$ stacks of cine magnetic resonance imaging (MRI) datasets. Inspired by this work, we present a pure 3D edge term, which is computed through the following strategy (Fig. 2): 1) estimation of the image edges (S) through a Canny edge detector (Canny, 1986), and 2) computation of the edge energy term $(\mathrm{Y})$ as an unsigned distance function between each volume point and the identified edges. The representation of $\mathrm{Y}$ as a distance function, improves the robustness of the edge detection strategy, filling small gaps due to missing edges in the map. Specifically, Eqs. (4) and (5) are now described as:

$F_{i}(\boldsymbol{y})=\lambda_{i}^{R} \cdot F^{R}(\boldsymbol{y})+\mathrm{Y}(\mathbf{y})$,

$\bar{g}_{i}\left(\boldsymbol{x}^{*}\right)=\bar{g}_{i}^{I M}\left(\boldsymbol{x}^{*}\right)=\lambda_{i}^{R} \cdot \overline{g^{R}}\left(\boldsymbol{x}^{*}\right)+\nabla_{r} \bar{Y}\left(\boldsymbol{x}^{*}\right)$,

with $\mathrm{Y}(\mathbf{y})=\min _{j}\left(\left\|\boldsymbol{x}-S_{j}\right\|\right), j$ representing each edge of the map $S$ and $\nabla_{r} \bar{Y}\left(\boldsymbol{x}^{*}\right)$ the edge energy map gradient along the radial direction, which is computed through centered finite differences. $\lambda_{i}^{R}$ is a positive hyper-parameter that balances the regional and edgebased terms.

\subsubsection{Regularization term}

In order to reduce the model's degrees of freedom and to prevent incorrect segmentation due to image artifacts, regularization terms are typically used. In the current work, we apply a curvature-based shape prior regularization to prevent concave shapes. As such, local regions with negative concavities are penalized, consequently pushing the surface outward. Specifically, a binary curvature-based switch is used:

$\overline{g^{C}}\left(\boldsymbol{x}^{*}\right)=-\mathcal{K}\left(\boldsymbol{x}^{*}\right) \cdot H\left(-\mathcal{K}\left(\boldsymbol{x}^{*}\right)\right)$,

updating Eq. (7) to:

$\bar{g}_{i}\left(\boldsymbol{x}^{*}\right)=\bar{g}_{i}^{I M}\left(\boldsymbol{x}^{*}\right)=\lambda_{i}^{R} \cdot \overline{g^{R}}\left(\boldsymbol{x}^{*}\right)+\nabla_{r} \bar{Y}\left(\boldsymbol{x}^{*}\right)+\lambda_{i}^{C} \cdot \overline{g^{C}}\left(\boldsymbol{x}^{*}\right)$.

where $\lambda_{i}^{R}$ represents positive hyper-parameters that balance the multiple terms used. $\mathcal{K}$ is the local curvature of the contour. Note that, $H\left(-\mathcal{K}\left(\boldsymbol{x}^{*}\right)\right)$ guarantees that only concave regions are regularized.

\subsubsection{BEAS-threshold}

BEAS-threshold was previously presented in Queirós et al. $(2014,2016)$ for initialization of a myocardial wall or aortic wall segmentation technique. The method uses a priori defined rules to locally expand or shrink the contour. The contour is updated by directly using the B-spline coefficients $\left(c_{i}[\boldsymbol{k}]\right)$ as described in Eqs. (3) and (9), allowing a fast evolution process with intrinsic control of the smoothness degree for each contour. The original BEAS-threshold method is purely based on intensities, using a fixed threshold (e.g. mean intensity of the target region) to generate a feature map to update the contours. Specifically, the fixed threshold is compared with the image intensity in each control point of 
the surface, and these points are then updated accordingly with the pre-defined rule (i.e. expand or shrink). No energy is used to control the contour evolution, being the process finished by simple factors, such as the number of iterations or the area. In this sense, Eqs. (3) and (9) are used to evolve the multiple contours, i.e. $i \in\{L A, R A, A O, L V, R V\}$, with:

$\overline{g^{R}}\left(\boldsymbol{x}^{*}\right)=\left\{\begin{array}{l}1, \text { if } \bar{I}\left(\boldsymbol{x}^{*}\right) \geq t h \\ -1, \text { otherwise }\end{array}\right.$,

where $\bar{I}\left(\boldsymbol{x}^{*}\right)$ is the image intensity at position $\boldsymbol{x}^{*}$, and th is the fixed threshold used. Both intensity and edge-based terms are now used to guide the initialization and make the strategy more suitable for heterogeneous regions. Moreover, it should be noticed that the ventricles are used in the current stage to define the valve plane, as explained in detail in Section 2.2.2.

\subsubsection{Contour refinement}

The contours refinement step directly minimizes the energy function in Eq. (2), using its derivative $(3,9)$. The strategy is used to refine the atrial region, assuming three independent contours, i.e. $i \in\{L A, R A, A O\}$. In opposition to the BEAS-threshold, the segmentation uses smaller steps computed using small portions of the image, consequently refining the contour to the real anatomy. The regional term $\left(F^{R}\right)$ is described using the localized signed Yezzi energy (Queirós et al., 2014):

$F^{R}(\boldsymbol{y})=u_{x}-v_{x}$

and its derivative $\overline{g^{R}}$ :

$\overline{g^{R}}\left(\boldsymbol{x}^{*}\right)=\left(\bar{I}\left(\boldsymbol{x}^{*}\right)-u_{x}\right)+\left(\bar{I}\left(\boldsymbol{x}^{*}\right)-v_{x}\right)$,

where $u_{x}$ and $v_{x}$ are the mean intensities inside and outside of the evolving interface at point $\boldsymbol{x}$, calculated using mask $B . \bar{I}\left(\boldsymbol{x}^{*}\right)$ is the image value at position $\boldsymbol{x}=\left\{x_{1}=\psi\left(\boldsymbol{x}^{*}\right), x_{1}, \ldots, x_{z}\right\}$. The presented energy searches for the optimal position as the maximum contrast point between regions. However, since all chambers are brighter than the cardiac wall, a signed version of this energy is used. As such, a specific representation of the target transition (bright to dark) is explicitly embedded into the functional energy, making it less sensitive to artifacts.

\subsection{Competitive B-spline explicit active surfaces}

The aforementioned free-evolution strategy (Section 2.1) allows segmenting multiple structures, but does not prevent overlapping regions, being therefore sub-optimal for several medical applications (e.g. evaluation of mid thin walls). In this section, we expand the previous methodology introducing a novel competitive strategy. This competitive strategy locally constrains the evolution of each contour when they are too near, preventing overlapping regions. Moreover, the novel competitive strategy is expanded to prevent merging between contours, allowing an accurate segmentation of mid thin walls as found in the atrial region. For the sake of clarity, we start by explaining how this competition can be performed between two contours (Section 2.2.1), being subsequently expanded for $n$ contours and specifically for the atrial region segmentation problem (Section 2.2.2). The authors would like to emphasize that no restrictions about the models' shapes are made. Moreover, the current methodology can be used for either 2D or 3D problems.

\subsubsection{Two contours}

Assuming a hypothetic segmentation scenario with two regions (e.g. LA and RA only), two independent functions and their corresponding evolution energies, $E_{1}$ and $E_{2}$, are required. The energy combination is performed using Eq. (2) and the energy minimized using Eq. (3). In order to include a competition strategy between the different contours, a novel term should be included in

$$
\begin{aligned}
& \text { Eq. (9): } \\
& \bar{g}_{1}\left(\boldsymbol{x}^{*}\right)=\bar{g}_{1}^{I M}\left(\boldsymbol{x}^{*}\right)+\alpha_{1 \rightarrow 2} \cdot \bar{g}_{1 \rightarrow 2}^{C P T}\left(\boldsymbol{x}^{*}\right) \text { and } \\
& \bar{g}_{2}\left(\boldsymbol{x}^{*}\right)=\bar{g}_{2}^{I M}\left(\boldsymbol{x}^{*}\right)+\alpha_{2 \rightarrow 1} \cdot \bar{g}_{2 \rightarrow 1}^{C P T}\left(\boldsymbol{x}^{*}\right), \text { with } \\
& \alpha_{2 \rightarrow 1}=1-\left|\alpha_{1 \rightarrow 2}\right|,
\end{aligned}
$$

where $\bar{g}^{I M}$ are the features extracted from the image (i.e. signed localized Yezzi, edge based and regularization terms) and $\bar{g}^{C P T}$ represents the competition term. Moreover, $\alpha_{1 \rightarrow 2}, \alpha_{2 \rightarrow 1} \in[-1 ; 1]$ are confidence terms. Both confidence terms are used to increase the influence of one of the contours throughout the competition process, due to a priori knowledge of superior image quality in one of the regions. $\alpha_{1 \rightarrow 2}=0$ means that no penalization related with competition is applied in contour 1 , and the contour evolution is only performed based on the image data. In contrast, total penalization $\left(\alpha_{2 \rightarrow 1}=1\right)$ is applied in contour 2 based on the competition process. $\alpha_{1 \rightarrow 2}=0.5$ represents an equal confidence on both contours. Moreover, it may be noted that $\alpha<0$ is required for concentric contours (i.e. contours with the same expansion/shrinking direction), where the penalization factor should be applied in opposing directions, increasing the wall thickness between them and preventing overlapping regions.

Regarding the competition term $\left(\bar{g}^{C P T}\right)$, it is described as:

$\bar{g}_{1 \rightarrow 2}^{C P T}\left(\boldsymbol{x}^{*}\right)=\left(R_{T}-\boldsymbol{\psi}^{1 \rightarrow 2}\right) \cdot H\left(R_{T}-\boldsymbol{\psi}^{1 \rightarrow 2}\right)$,

$\bar{g}_{2 \rightarrow 1}^{C P T}\left(\boldsymbol{x}^{*}\right)=\left(R_{T}-\boldsymbol{\psi}^{2 \rightarrow 1}\right) \cdot H\left(R_{T}-\boldsymbol{\psi}^{2 \rightarrow 1}\right)$,

with $R_{T}$ representing the estimated minimal thickness parameter, $\psi$ is a signed distance map between each node of the surface 1 against the entire surface 2 (and vice-versa), and $H$ a Heaviside operator. Note that $H$ is only equal to one in nodes with $\psi$ lower than $R_{T}$, being zero in the remaining nodes (Fig. 3). Therefore, the interactive strategy is only applied in the nearest regions of the contours (i.e. distance lower than $R_{T}$ ). Regarding $\psi$, it is computed as the minimal result of three tested possibilities: 1) a point-to-point distance between all the vertices of both surfaces; 2) an edge-topoint distance defined as the intersection result between all the edges of surface 2 against the target point in surface 1 ; and 3 ) a face-to-point distance, computed as the intersection between the multiple faces/planes of surface 2 against the point in surface 1 . It should be noticed that overlapping contour regions are defined as negative distances. The reader is kindly directed to Baerentzen and Aanaes (2005) for further details on signed distance function computation.

A schematic about the competition workflow can be found in Fig. 3. As described above, the distance between two surfaces is used to locally constrain the surfaces' evolution. When the distance between contours is lower than $R_{T}$, a force with opposite direction to the contour evolution direction is applied to prevent fast steps, consequently allowing small refinements of both contours (without changing force orientation, Fig. 3b). Although a minimal thickness parameter $\left(R_{T}\right)$ is defined a priori, the final segmentation result with thinner mid-walls between contours (i.e. minimal distance lower than $R_{T}$ ) is allowed, consequently increasing the flexibility of the model to abnormal situations. Nevertheless, when the thickness between both contours is too small, high penalization is applied requiring both contours to retreat (Fig. 3c). Then, the minimization continues and correct refinement to the middle wall is possible.

\subsubsection{Expansion to n-contours}

For $n$ contours (and specifically for atrial region segmentation, i.e. LA, RA, Ao), a total of $n$ energies ( $n=3$ for the atrial region) are now required to control each contour as described in Section 


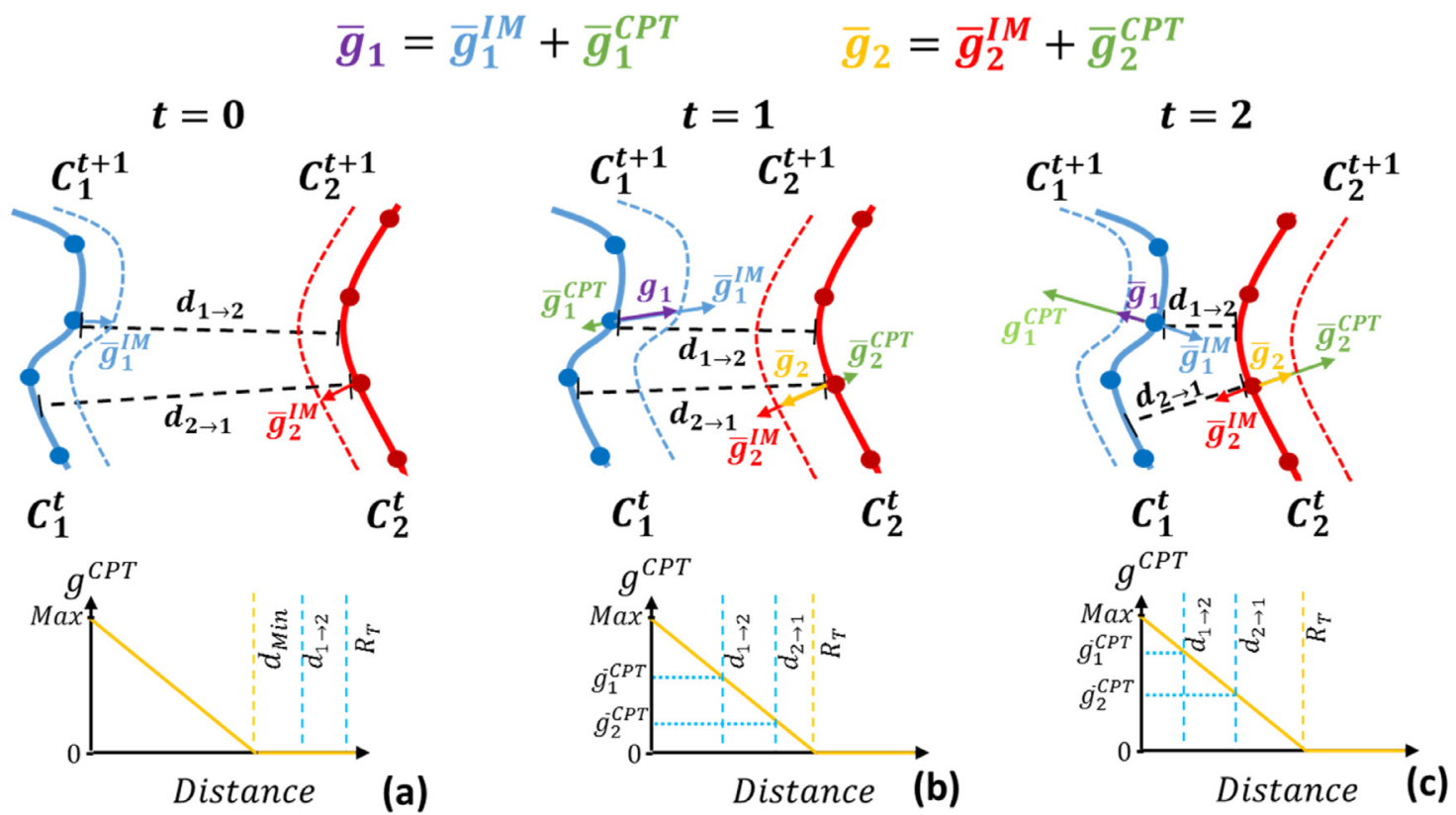

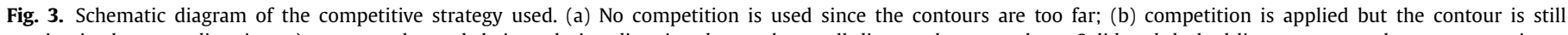

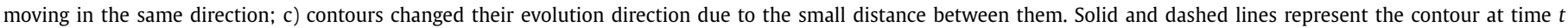
and $t+1$, respectively.

2.1.1. Contrary to Section 2.2.1, multiple competitions between the $n$ contours are now considered requiring a generalization of Eqs. (13) and (14) to:

$\bar{g}_{i}\left(\boldsymbol{x}^{*}\right)=\bar{g}_{i}^{I M}\left(\boldsymbol{x}^{*}\right)+\sum_{m=1, m \neq i}^{n} \alpha_{i \rightarrow m} \cdot \bar{g}_{i \rightarrow m}^{C P T}\left(\boldsymbol{x}^{*}\right)$,

$\alpha_{i \rightarrow m, i \neq m}=\left(1-\left|\alpha_{m \rightarrow i, i \neq m}\right|\right)$,

with,

$\bar{g}_{i \rightarrow m, i \neq m}^{C P T}\left(\boldsymbol{x}^{*}\right)=\left(R_{T}-\boldsymbol{\psi}^{i \rightarrow m}\right) \cdot H\left(R_{T}-\boldsymbol{\psi}^{i \rightarrow m}\right)$.

Note that Eq. (17) combines all the $n$ contour pairs. As such, in each iteration, the distance between all pairs is computed and all the thin regions refined through the competition between the nearest contours.

The proposed competitive implementation is integrated on both the BEAS-threshold (Section 2.1.4) and contour refinement stages (Section 2.1.5). During the BEAS-threshold, the competitive strategy is used to: 1) improve the LA-RA-AO contours initialization thanks to the spatial interactions between them; and 2) define the valve plane through the competition between atria and ventricles. Contrarily, in the contour refinement stage, the LA-RA-AO contours compete between them to accurately identify the atrial boundaries. The ventricles are not refined throughout the segmentation due to the RV anatomy and the difficulty in describing it using an explicit function.

In Appendix $A$, the mathematical formalism required to expand the proposed competitive strategy for a traditional level-set model is presented, showing the versatility of the described methodology.

\section{Experiments}

Three experimental scenarios are used to prove the advantages of the proposed competitive BEAS for atrial segmentation, namely atrial region evaluation in CT (Section 3.1), LA benchmark in CT (Section 3.2) and atrial region evaluation in MR (Section 3.3).

Two major comparisons are addressed: 1) differences between the competitive BEAS against the free-evolution strategy, and 2) comparison between the competitive strategy against state-of-theart methods. For each experiment, one observer was responsible
Table 1

Acquisition parameters of the CT scanners.

\begin{tabular}{lll}
\hline Parameter & Brilliance CT and Brilliance iCT & SOMATOM force \\
\hline Manufacter & Philips & Siemens \\
No. of patients & 30 & 41 \\
Detector rows & $16-, 40-, 64-$ and 256-slices & 64,128 slices \\
Contrast injection & $40-100 \mathrm{ml}$ & $40-100$ \\
Image resolution $\left(\mathrm{mm}^{2}\right)$ & $0.30 \times 0.30$ to $0.78 \times 0.78$ & $0.69 \times 0.69$ \\
Slice thickness & 0.33 to $1.00 \mathrm{~mm}$ & 0.8 \\
Matrix size & $512 \times 512$ & $512 \times 512$ \\
No. of phases & 1 & 1 \\
\hline
\end{tabular}

for identifying the points required to initialize the method through one click in each chamber and three clicks in the aortic tract.

\subsection{Atrial region assessment using $C T$}

\subsubsection{Description}

Forty-one datasets retrospectively obtained in the clinical practice from patients with suspicion of pathology in the atrial region were used to validate the proposed competitive approach. Specifically, patients with pacemaker, mitral and aortic prosthetic valves, atrial body enlargement and candidates to pulmonary vein ablation were included. ECG-gated cardiac multi-slice CT images were acquired with multi-detector Siemens CT scanner, Table 1. Both enddiastolic and end-systolic phases were included.

\subsubsection{Ground truth generation}

The LA and RA segmentation was performed by one expert using the semi-automatic CARTO3 segmentation tool (Biosense Webster, Diamond Bar, CA, USA), followed by manual corrections. More details about the segmentation can be obtained in Bourier et al. (2016). In order to identify the atrial body and similarly to the proposed in Tobon-Gomez et al. (2015), multiple bounding boxes were generated around the pulmonary veins, vena cava and left and right atrial appendage (LAA and RAA). All bounding boxes were drawn around the ostia of each structure. ParaView (Kitware, Inc.) (Squillacote and Ahrens, 2007) was used to visualize the sur- 
Table 2

Parameters used to define the competition between surface $\alpha_{S 1 \rightarrow S 2}$.

\begin{tabular}{|c|c|c|c|c|c|c|c|c|c|}
\hline & S2 & & & & & & & & \\
\hline \multirow[t]{7}{*}{ S1 } & Chambers & \multicolumn{5}{|c|}{ Initialization } & \multicolumn{3}{|c|}{ Segmentation } \\
\hline & & LA & RA & AO & LV & RV & LA & RA & $\mathrm{AO}$ \\
\hline & LA & - & 0 & 0.5 & 0.5 & 0 & - & 0.5 & 0.5 \\
\hline & RA & 1 & - & 1 & 1 & 0.5 & 0.5 & - & 0.5 \\
\hline & $\mathrm{AO}$ & 0.5 & 0 & - & 0.5 & 0 & 0.5 & 0.5 & - \\
\hline & LV & 0.5 & 0 & 0.5 & - & 0 & - & - & - \\
\hline & RV & 1 & 0.5 & 1 & 1 & - & - & - & - \\
\hline
\end{tabular}

LA - Left atrium; RA - Right atrium; AO - Aorta; LV - Left ventricle; RV Right Ventricle.

face generated by the expert, and to identify the multiple bounding boxes. Mitral valve and tricuspid valve planes were obtained through the following steps: 1) manual delineation of the valve region in several rotated slices, and 2) plane fitting using all scattered points. Furthermore, aortic tract manual delineation was performed through an in-house framework (Queirós et al., 2016). Initially, three points along the aortic tract were manually defined to generate a centerline. Then, several planes (25-30 planes) perpendicular to the centerline were used to perform multiple manual 2D delineations. Finally, all scattered points were transformed into a 3D surface. Of note, all bounding boxes and planes were only used to evaluate the segmentation performance, not interfering in the segmentation pipeline.

\subsubsection{Implementation details}

The Canny edge detector was computed using a sigma of $1.5 \mathrm{~mm}$, and a lower and upper threshold of 0.7 and 0.9 , respectively. $\lambda_{i}^{R}$ was set to 0.5 during the initialization, and $\lambda_{i}^{R}=20$ in segmentation. Regularization through curvature analysis $\left(\lambda_{i}^{C}=20\right)$ was only applied in the RA, RV and LV. Regarding the competition, Table 2 presents the parameters used and a $R_{T}$ of $2 \mathrm{~mm}$ was employed as suggested in Beinart et al. (2011). It may be noted that, during the initialization, the left heart controls the right heart evolution, due to the superior contrast and reduced number of artifacts usually found in the left heart compared to the right side. In other words, during the BEAS-threshold, the left side contours evolve freely pushing the right heart contours. Then, during the segmentation, competitive contours with equal weights $(\alpha=0.5)$ were used. The fixed threshold (th) was computed as the average value between the mean intensity on the selected region (window of size equal to $3 \times 3 \times 3 \mathrm{~mm}^{3}$ ) and the expected intensity of the atrial/aortic walls ( $50 \mathrm{HU}$, (Ecabert et al., 2008)). The stop criteria of the BEAS-threshold method relies on the difference between mesh positions in two consecutive iterations and it finishes when small differences are found. Regarding the BEAS parameters, a total of $40 \times 40$ points were used to represent each contour. Local profiles with $10 \mathrm{~mm}$ inward and outward of the contour were used, and $h_{r_{i}}=h_{s_{i}}=1$ for $i \in\{L A, R A, L V, R V\}$ and $h_{r_{i}}=h_{s_{i}}=2$ for $i \in\{A O\}$ were applied.

\subsubsection{Statistical analysis}

The absolute point-to-surface (P2S) distance, Dice coefficient (DC) and 95th percentile of Hausdorff distance were computed for each chamber (LA, RA and Ao) to compare the methodology with and without competition. The influence of the virtually generated bounding boxes and mitral/tricuspid valve planes was also assessed. A paired $t$-test $(p<0.05)$ between the strategy with or without competition was used to check for statistically significant differences on the results. Furthermore, a small region of interest (ROI) was created around each thin wall (aortic and atrial wall) in order to assess the segmentation accuracy in these regions. The region was defined as the largest connected component with a thickness inferior to $5 \mathrm{~mm}$ (computed as LA vs RA, LA versus AO, RA versus Ao). The errors obtained with competition and without competition were assessed through P2S, 95th percentile of Hausdorff distance and a Wilcoxon matched-pair test to check for statistically significant differences $(p<0.05)$. The influence of the estimated thickness parameter $R_{T}$ on the final segmentation result was also assessed and compared through a paired $t$-test $(p<0.05)$ to check for statistically significant differences. Finally, the computational time of both approaches (with and without competition) was registered. All results were computed using MATLAB code (no parallelization) on an Intel (R) i7 CPU at $2.8 \mathrm{GHz}$ and $16 \mathrm{~GB}$ of RAM. $\mathrm{A} \mathrm{C}++$ implementation of the competition strategy was wrapped in the MATLAB Code.

\subsubsection{Results}

Table 3 presents the results obtained for both methodologies in terms of P2S, DC and Hausdorff distance. The RA presented the highest P2S error with $1.68 \pm 0.47 \mathrm{~mm}$ and the aortic tract the lowest with an error of $0.65 \pm 0.12 \mathrm{~mm}$. Moreover, when comparing the competitive strategy and its free-evolution version, a superior performance was always achieved by the competitive version for all the assessed regions (Fig. 4). Fig. 5 presents the errors obtained when a small ROI around the aortic/atrial walls is assessed, where a clear advantage of the proposed competitive technique is observed. Globally, the strategy with competition showed a statistically significant superior accuracy when compared with the free-evolution version. The former strategy is however more computationally demanding, requiring $73.4 \pm 6.9 \mathrm{~s}$ per dataset, against the $35.8 \pm 3.7 \mathrm{~s}$ recorded for the free-evolution approach. Representative segmentation cases corresponding to the 10th, 30th, 50th, 70th, and 90th percentiles according to the average P2S error are shown in Fig. 6. Moreover, results in patients with anatomical pathologies are presented in Fig. 7. Finally, the influence of the $R_{T}$ parameter throughout the competitive strategy is shown in Fig. $8 \mathrm{a}$. No significant differences were observed between the selected value (i.e. $2 \mathrm{~mm}$ ) and its neighbors, but in contrast, statistically significant differences were found when too high values were used.

\subsection{Left atrium assessment using $C T$}

\subsubsection{Description}

Recently, a benchmark (STACOM 2013) was published to assess the accuracy of left atrium segmentation techniques. The public database has thirty datasets, 10 datasets for training and 20 datasets for testing. Only LA segmentation methods are allowed to be evaluated with the current benchmark. Further details about acquisition and ground truth generation are indicated in Table 1 and in Tobon-Gomez et al. (2015).

\subsubsection{Statistical analysis}

The segmentation accuracy was assessed using two metrics described in the original manuscript: P2S and DC. Furthermore, the obtained result with the proposed methodology (with and without competition) was compared with the 5 best works assessed in the current benchmark (total of 9), namely a region growing formulation with rough contour initialization using an atlasbased approach (LTSI-VRG) (Sandoval et al., 2013), a probabilistic atlas approach (LUB-SRG) proposed by Stender et al. (2013), a marginal space learning strategy with (SIE-MRG) (Tobon-Gomez et al., 2015) or without refinement (SIE-PMB) (Zheng et al., 2008) through graph-cuts, and a multi-atlas approach with global and local transforms for LA segmentation only (UCL-1C) (Zuluaga et al., 2013). Inter-observer variability is also available. Furthermore, the added value of the proposed approach was explored, through a comparison of the result obtained with and without competition using an unpaired $t$-test $(p<0.05)$. 
Table 3

Point-to-surface (P2S) error, Dice coefficient and 95th percentile Hausdorff distance obtained between the semiautomatic method (with and without competition) against the manual delineation in CT. The left atrium and right atrium body are assessed. Aortic tract errors are also analyzed.

\begin{tabular}{|c|c|c|c|c|c|c|c|}
\hline \multirow[b]{2}{*}{ Competition } & & \multicolumn{2}{|l|}{$\mathrm{P} 2 \mathrm{~S}(\mathrm{~mm})$} & \multicolumn{2}{|l|}{ Dice } & \multicolumn{2}{|c|}{ Hausdorff (mm) } \\
\hline & & With & Without & With & Without & With & Without \\
\hline \multirow[t]{4}{*}{ Left Atrium } & Entire & $1.54 \pm 0.32^{*}$ & $1.57 \pm 0.33$ & $0.91 \pm 0.01$ & $0.92 \pm 0.01$ & $6.52 \pm 2.41^{*}$ & $6.67 \pm 2.42$ \\
\hline & MV & $1.36 \pm 0.27 *$ & $1.39 \pm 0.28$ & $0.93 \pm 0.01$ & $0.93 \pm 0.01$ & $5.46 \pm 1.87^{*}$ & $5.55 \pm 1.86$ \\
\hline & PV & $1.20 \pm 0.20$ & $1.25 \pm 0.23$ & $0.94 \pm 0.01$ & $0.94 \pm 0.01$ & $4.47 \pm 1.63$ & $4.85 \pm 1.75$ \\
\hline & LAA & $1.07 \pm 0.17$ & $1.14 \pm 0.21$ & $0.95 \pm 0.01$ & $0.95 \pm 0.01$ & $3.36 \pm 0.98$ & $3.62 \pm 1.10$ \\
\hline \multirow[t]{4}{*}{ Right Atrium } & Entire & $2.13 \pm 0.51^{*}$ & $2.37 \pm 0.66$ & $0.87 \pm 0.03 *$ & $0.85 \pm 0.05$ & $8.13 \pm 2.18^{*}$ & $8.90 \pm 2.47$ \\
\hline & TV & $2.01 \pm 0.46^{*}$ & $2.26 \pm 0.68$ & $0.88 \pm 0.03^{*}$ & $0.87 \pm 0.05$ & $7.81 \pm 1.97^{*}$ & $8.54 \pm 2.48$ \\
\hline & VC & $1.87 \pm 0.47^{*}$ & $2.13 \pm 0.67$ & $0.90 \pm 0.02 *$ & $0.89 \pm 0.05$ & $7.20 \pm 2.03^{*}$ & $8.14 \pm 2.63$ \\
\hline & RAA & $1.68 \pm 0.47^{*}$ & $1.98 \pm 0.72$ & $0.91 \pm 0.02 *$ & $0.90 \pm 0.05$ & $6.03 \pm 2.04 *$ & $7.52 \pm 3.57$ \\
\hline Aortic tract & Entire & $0.65 \pm 0.12^{*}$ & $0.68 \pm 0.13$ & $0.94 \pm 0.02$ & $0.94 \pm 0.02$ & $1.61 \pm 0.33$ & $1.67 \pm 0.36$ \\
\hline
\end{tabular}

* Paired $t$-test between the result obtained with and without competition $(p<0.05)$. MV - Mitral Valve; PV - Pulmonary Veins; LAA - Left Atrial Appendage; TV - Tricuspid valve; VC - Vena Cava; RAA - Right Atrial Appendage.
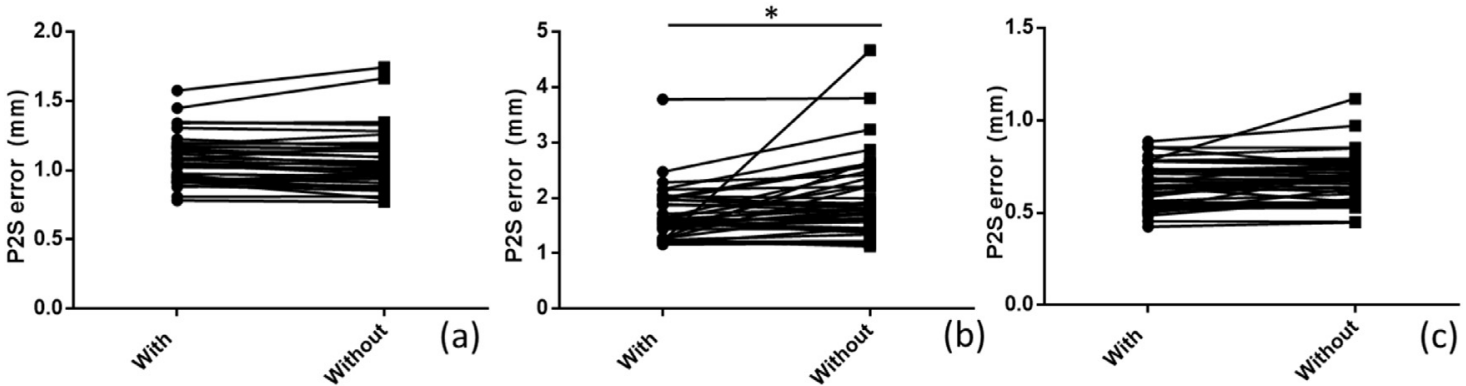

* - Wilcoxon matched-pair between with and without competition $(p<0.05)$;

Fig. 4. Error obtained with and without competition for the LA (a), RA (b) and aortic tract (c) in CT.
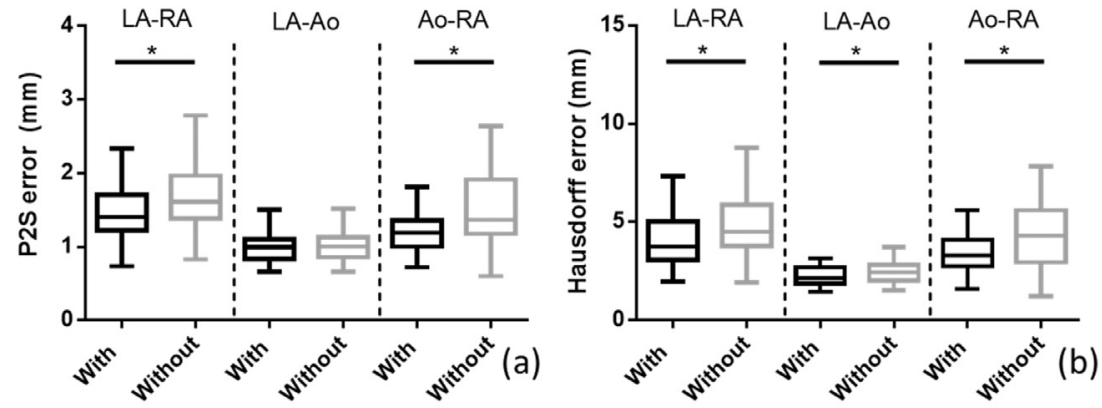

${ }^{\star}$ Paired t-test between with and without competition $(p<0.05)$.

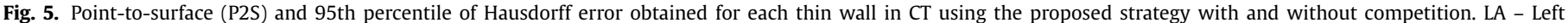
atrium, RA - Right Atrium, Ao - Aortic tract.

\subsubsection{Results}

Fig. 9 shows a comparison between the proposed methodology (competitive BEAS) and the state-of-the-art techniques for LA body segmentation. The proposed strategy obtained a P2S error of $0.89 \pm 1.03$ (with competition), proving its robustness and accuracy with results similar to the remaining techniques. Regarding the competitive strategy, statistically significant differences $(p=0.02)$ were found against the free-evolution technique in terms of P2S error.

\subsection{Atrial region assessment using MRI}

\subsubsection{Description}

Similarly to Section 3.2, the current data was obtained from an available benchmark (Tobon-Gomez et al., 2015). Again, 30 datasets were acquired, 10 datasets are used as training, while the remaining 20 cases are used for the algorithm evaluation. The reader is kindly directed to Tobon-Gomez et al. (2015) for further details about the image acquisition protocol.

\subsubsection{Ground truth generation}

The LA was segmented using the strategy described in TobonGomez et al. (2015). Contrarily to the CT data (Section 3.2), no limitations were imposed on these datasets, allowing their application for further studies. In this sense, and in order to assess the accuracy of the competitive strategy in a different imaging modality, a manual contouring of the RA and the aortic tract was additionally performed. The RA was segmented using the MITK software (Medical Imaging Interaction Toolkit, (Wolf et al., 2005)), where multiple 2D slices were delineated and then interpolated to a 3D surface. Moreover, bounding boxes and valve plane were generated as explained in Section 3.1. Regarding the aortic tract, it was segmented as described in 3.1 .

\subsubsection{Implementation details}

Due to the particularities of the MRI datasets (i.e. noisy images, with superior pixel spacing when compared with a CT acquisition), small modifications were required in specific parameters of the segmentation methodology, namely: 1) $\lambda_{i}^{R}$ was set to 


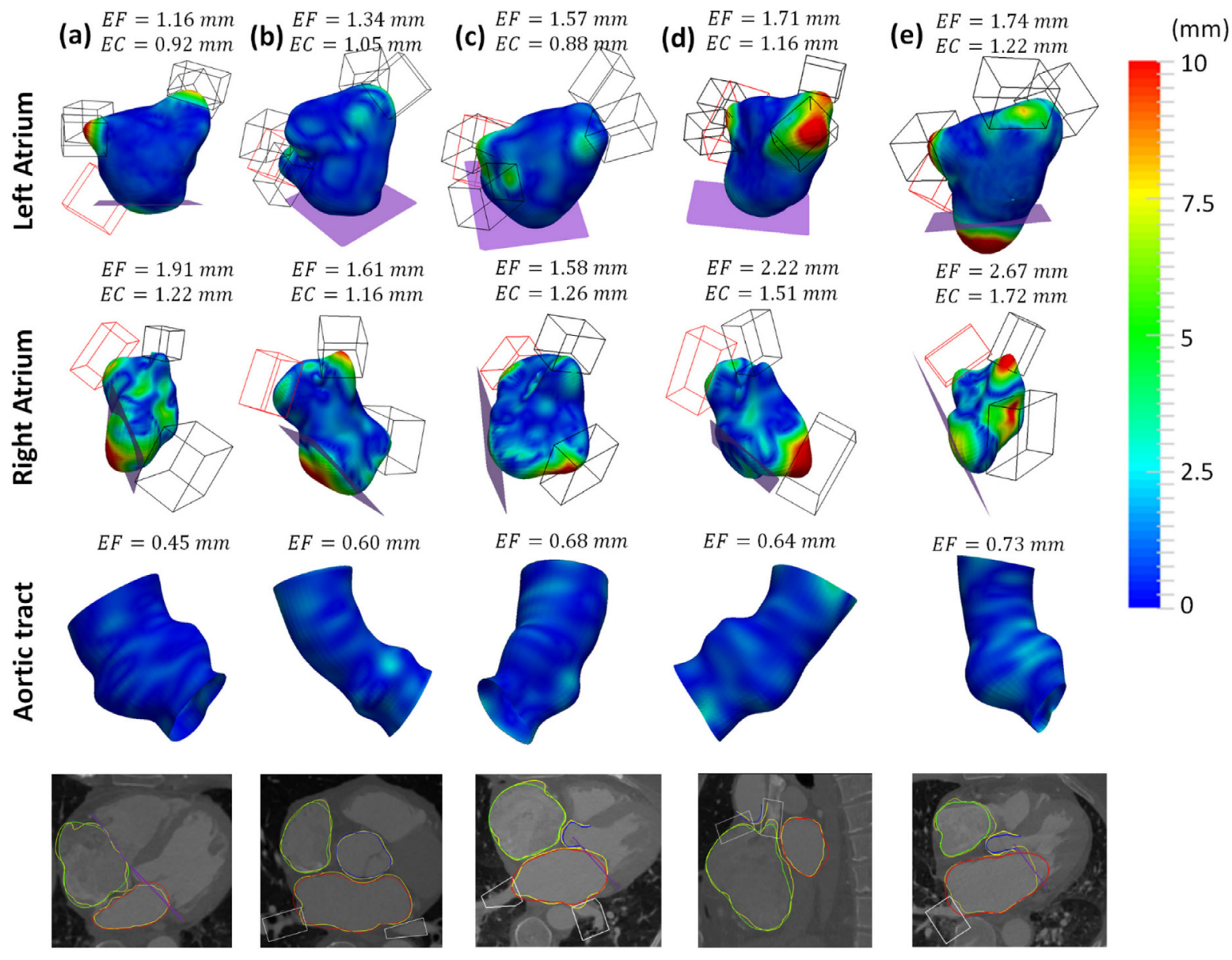

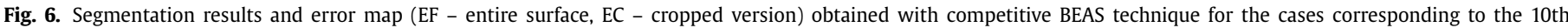

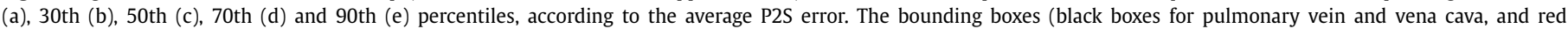

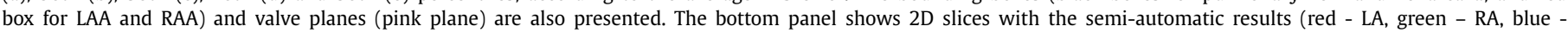

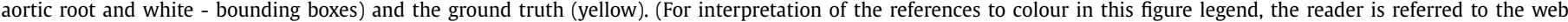
version of this article.)

1 during the threshold stage; 2 ) each contour was discretized by $32 \times 32$ points; and 3) $h_{r_{i}}=1, h_{s_{i}}=2$ for $i \in\{L A, R A\}$. The optimal parameters were estimated using the training datasets only.

\subsubsection{Statistical analysis}

The LA body segmentation was assessed in terms of P2S error and DC using the software released with the benchmark. Moreover, the result obtained with the proposed methodologies was compared with the 5 best strategies described in Tobon-Gomez et al. (2015) (a total of 8 were presented). In detail, the multi-atlas approach described by Zuluaga et al. (2013) with an atlas database encompassing all cardiac cavities (UCL4C) or LA only (UCL1C) and the 2D segmentation strategy with threshold-based techniques and circularity shape descriptors (TLEMCEN) (Ammar et al., 2013) were included. Furthermore, two versions of the strategy proposed in Sandoval et al. (2013) were also considered, one with label fusion through majority voting (LTSI-VRG) and another using the STAPLE (LTSI-VSRG) algorithm. Inter-observer variability is also available. The RA and aortic tract were assessed in terms of P2S, DC and 95th percentile of Hausdorff distance. A paired $t$-test $(p<0.05)$ was computed to check for statistically significant differences between the technique with and without competition. Similarly to Section 3.1, the errors found at aortic/atrial wall were assessed using the P2S error, the 95th percentile of Hausdorff and Wilcoxon matchedpair ranked test $(p<0.05)$. Furthermore, the influence of the $R_{T}$ parameter on the atrial segmentation in MRI was also evaluated. Fi- nally, the computational time required by each methodology was recorded.

\subsubsection{Results}

Fig. 10 presents the results obtained by the proposed technique for LA segmentation using the benchmark. The proposed strategy obtained a mean error of $1.57 \mathrm{~mm}$, proving to be similar against the remaining strategies available in literature. A similar performance was obtained by the proposed method when compared with the inter-observer variability. Fig. 11 presents the results in each individual dataset, proving the clear advantages of the competitive strategy (with statistically significant differences, $p=0.04$ ) against the traditional BEAS formulation. Regarding the remaining chambers, Table 4 indicates the accuracy of each semi-automatic contouring against the manual one. The influence of each region (RAA, vena cava, valve plane) in the final contour was also assessed, showing a correct and accurate definition of the RA body and aortic tract. Again, the competitive strategy proved to be more robust than the free evolution technique, with statistically significant differences. Specifically, the RA proved to benefit most from the proposed competition formulation. Looking for the wall region analysis, a correct definition of these smooth and thin regions was only achieved by the competitive BEAS (Fig. 12). Furthermore, the sensitivity of the segmentation result for different $R_{T}$ thickness parameters was evaluated (see Fig. 8b), presenting a similar trend to the one obtained for CT datasets. Regarding the computational 

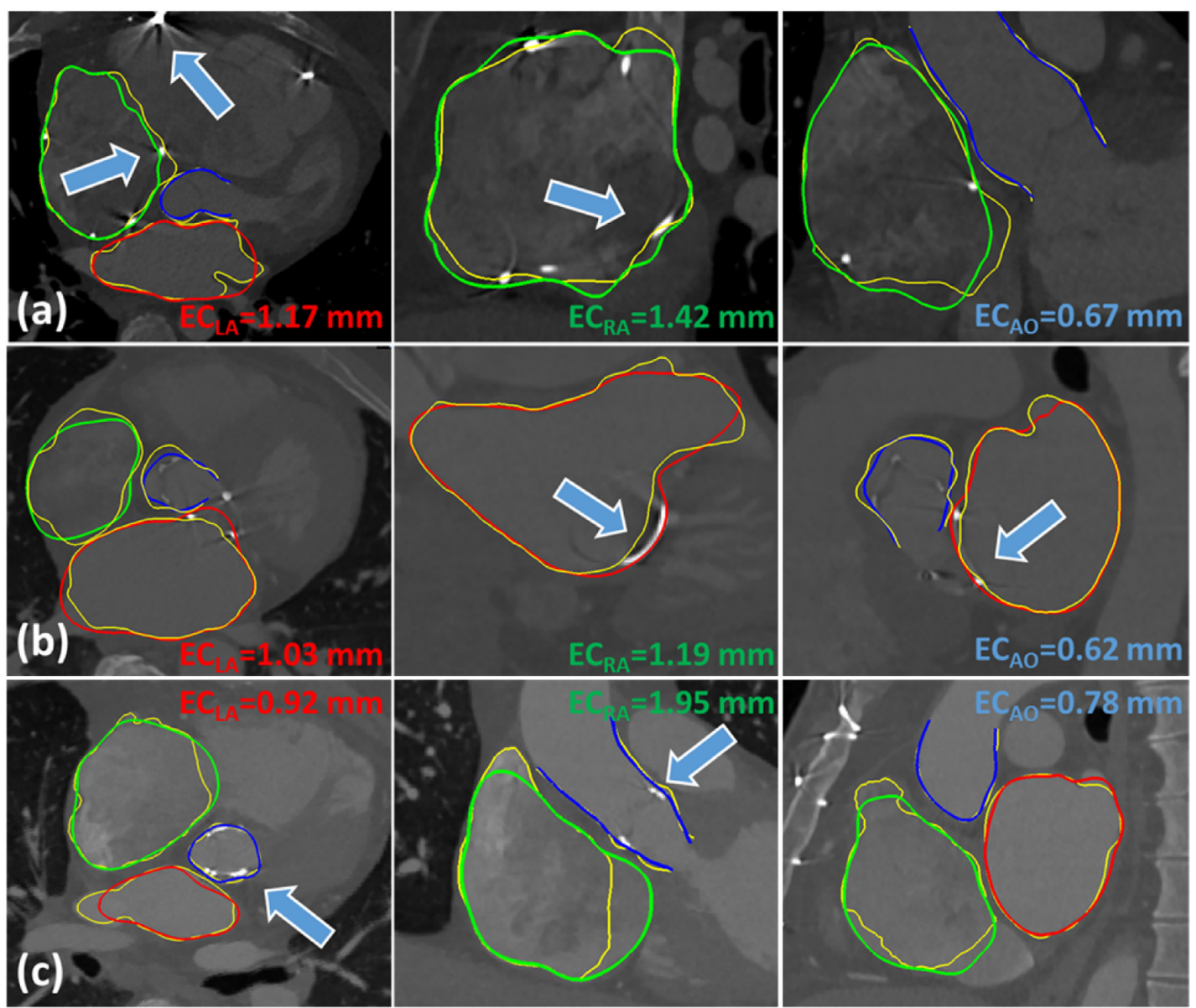

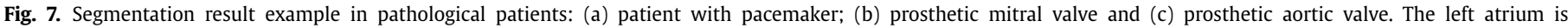

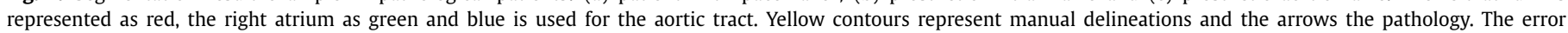

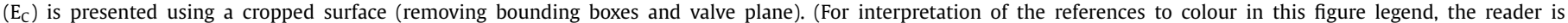
referred to the web version of this article.)

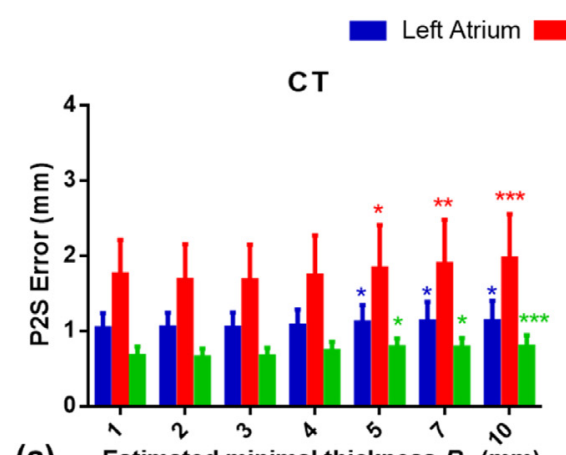

(a) Estimated minimal thickness $R_{T}(\mathrm{~mm})$

Right Atrium Aorta

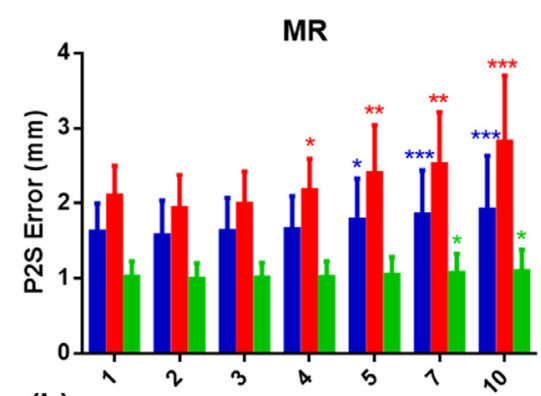

(b) Estimated minimal thickness $R_{T}(\mathrm{~mm})$

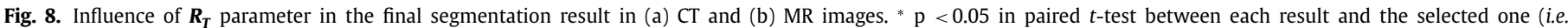
$2 \mathrm{~mm})$.

time, the competitive BEAS required $28.9 \pm 6.2 \mathrm{~s}$, while the traditional BEAS only needed $16.0 \pm 1.4 \mathrm{~s}$. Representative example results are presented in Fig. 13.

\section{Discussion}

In this study, we present a novel technique to accurately segment multiple structures. In opposition to the majority of the state-of-the-art techniques, constraints to allow thin walls between multiple structures are embedded. Furthermore, when compared with previous works addressing the same issue (Gao et al., 2012), the proposed formulation appears to present a superior performance for the delineation of heterogeneous and noisy walls, keeping a minimal wall thickness for all the different scenarios. This technique was integrated into an efficient 3D segmentation framework and the advantages of the novel competitive methodology 
LTSI_VRG - (Sandoval et al., 2013); LUB_SRG - (Stender et al., 2013);

SIE_PMB and SIE_MRG - (Zheng et al., 2008); UCL_1C - (Zuluaga et al., 2013)

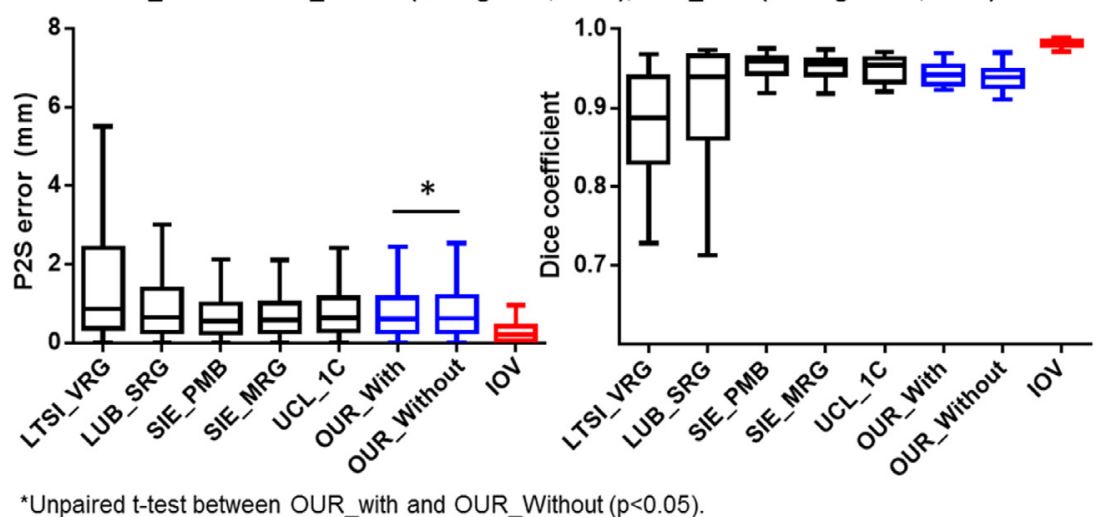

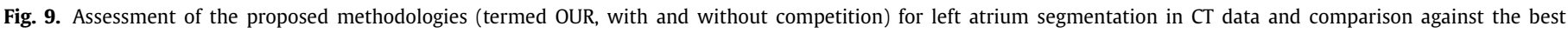
results available in the left atrium benchmark. The team's name were defined based on [34]. IOV represents the inter-observer variability.

LTSI_VRG and LTSI_VSRG - (Sandoval et al., 2013);

TLEMCEN - (Ammar et al., 2013); UCL_1C and UCL_4C - (Zuluaga et al., 2013)

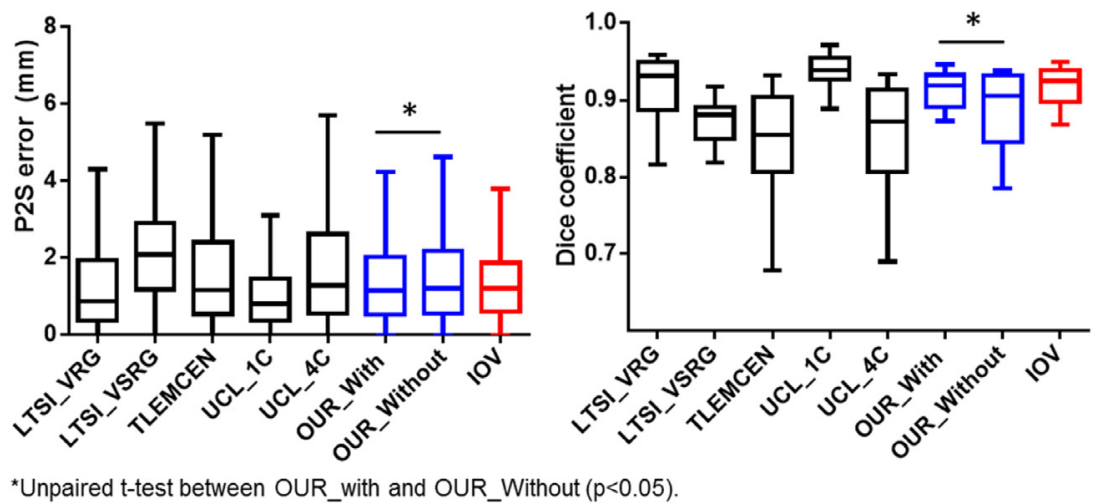

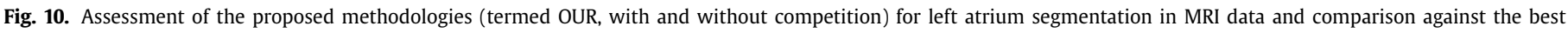
results available in the left atrium benchmark. The team's name were defined based on [34]. IOV represents the inter-observer variability.
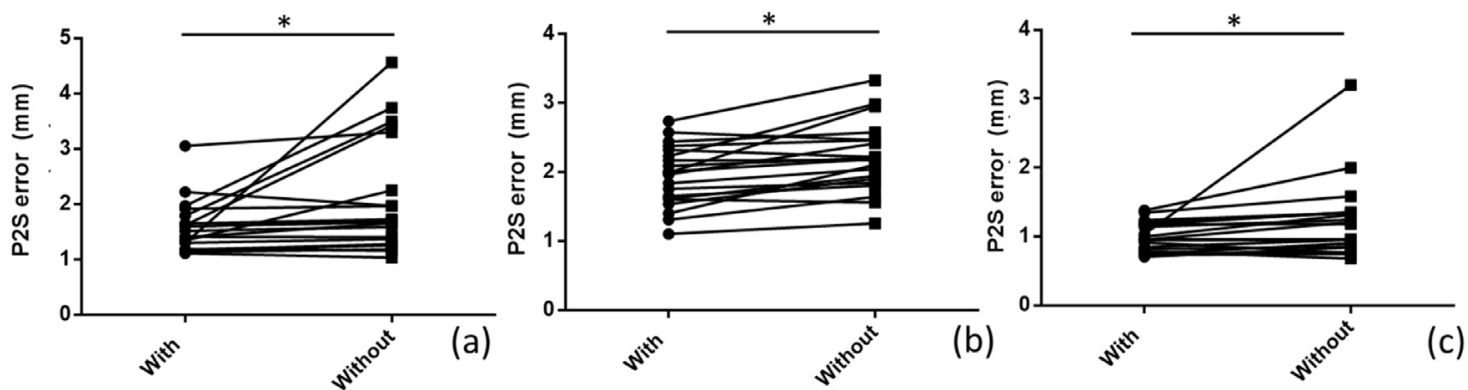

* - Wilcoxon matched-pair between with and without competition $(p<0.05)$;

Fig. 11. Point-to-surface error obtained with and without competition for the LA (a), RA (b) and aortic tract (c) in MRI.

Table 4

Point-to-surface (P2S) error, Dice coefficient and 95th percentile of Hausdorff distance obtained between the semiautomatic method (with and without competition) against the manual delineation in MR. The right atrium body and aortic tract error are assessed.

\begin{tabular}{llllllll}
\hline \multirow{2}{*}{ Competition } & & P2S $(\mathrm{mm})$ & & DICE & & \multicolumn{2}{l}{ Hausdorff (mm) } \\
& & With & Without & With & Without & With & Without \\
\hline \multirow{2}{*}{ Right Atrium } & Entire & $2.74 \pm 0.63^{*}$ & $2.98 \pm 0.61$ & $0.83 \pm 0.04^{*}$ & $0.80 \pm 0.05$ & $10.76 \pm 2.80$ & $11.39 \pm 2.56$ \\
& TV & $2.66 \pm 0.65^{*}$ & $2.91 \pm 0.64$ & $0.84 \pm 0.04^{*}$ & $0.83 \pm 0.05$ & $10.38 \pm 2.90$ & $10.96 \pm 2.55$ \\
& VC & $2.26 \pm 0.49^{*}$ & $2.49 \pm 0.51$ & $0.88 \pm 0.03^{*}$ & $0.87 \pm 0.04$ & $8.64 \pm 2.48^{*}$ & $9.42 \pm 2.19$ \\
\multirow{2}{*}{ Aortic tract } & RAA & $1.94 \pm 0.44^{*}$ & $2.20 \pm 0.51$ & $0.90 \pm 0.03^{*}$ & $0.88 \pm 0.04$ & $6.54 \pm 1.97^{*}$ & $7.71 \pm 2.11$ \\
& Entire & $0.99 \pm 0.21^{*}$ & $1.21 \pm 0.58$ & $0.88 \pm 0.04^{*}$ & $0.86 \pm 0.07$ & $2.52 \pm 0.66^{*}$ & $3.11 \pm 2.12$ \\
\hline
\end{tabular}

* Paired $t$-test against zero $(p<0.05)$. TV - Tricuspid Valve; VC - Vena Cava; RAA - Right Atrial Appendage. 

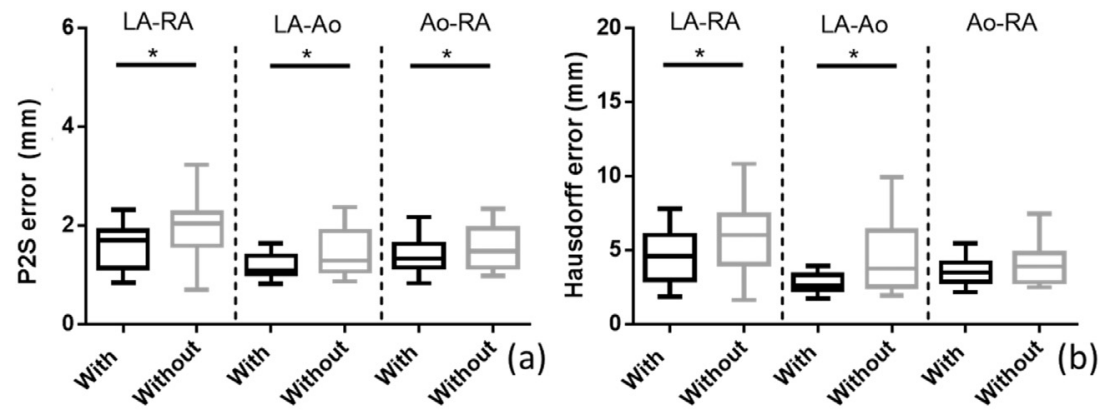

*Paired t-test between with and without competition $(p<0.05)$.

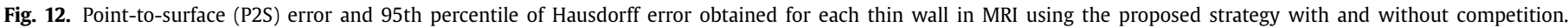
LA - Left Atrium, RA - Right atrium, Ao - Aortic tract.

was proven for atrial body segmentation. Note that evaluation of the mid thin walls is relevant in several clinical evaluations, such as optimal inter-atrial puncture location for transseptal puncture (Morais et al., 2017) and for the evaluation of the aortic wall thickness (Malayeri et al., 2008). To the author's best knowledge, no previous work was presented for accurate segmentation of the atrial region with intact mid-thin walls, being a clear novelty of this work. Previous works as Ecabert et al. (2011) and Zuluaga et al. (2013) simply merge the different contours (if overlap happens) or prevented gap/vacuum regions, being sub-optimal strategies for clinical evaluation of these thin regions. Although no significant differences are expected between the merged contour strategies and our approach in terms of segmentation evaluation metrics (e.g. P2S or Dice), the merged contour approaches are not suitable for novel image-guided minimally invasive interventions focused on atrial wall as presented by Bourier et al. (2016).

A first evaluation of the proposed competitive approach was performed on atrial region segmentation in CT images. The obtained results proved the high accuracy of the method, with errors similar to the ones found in other studies in literature (Ecabert et al., 2011; Kirişli et al., 2010; Zheng et al., 2008), particularly for LA (Fig. 9) and aortic tract (Table 3). Indeed, the proposed double-stage segmentation approach (i.e. BEAS-threshold - Section 2.1.4 and BEAS-segmentation - Section 2.1.5) showed its addedvalue and robustness for atrial segmentation. While the thresholdbased stage uses a fast contour growing approach to obtain a rough estimation of the target anatomy, the segmentation stage applies a localized/regional approach to accurately refine the contour to the real anatomic boundaries. Both stages are boosted by the edgebased and regularization terms, which always pushes the contour to the nearest edge (i.e. potential optimal transition) and prevents incorrect shapes caused by vacuum regions or noise (e.g. right atrium), respectively. It should be noted that pure segmentation without an initialization stage is not feasible, since the current initialization (i.e. sphere in the middle of the cavity) is too far from the real boundaries being therefore sensitive to local minima or even noisy regions inside the blood pool. Moreover, the proposed method showed its high and superior performance for segmentation of mid thin walls due to the novel proposed competitive strategy (Fig. 5). Since the current experiment was only focused on the atrial body and the effectiveness of the competitive approach for atrial region segmentation, 3D bounding boxes were virtually generated by one experienced observer to separate the atrial body from the great vessels. Similar strategies to compute the segmentation errors have been presented in literature (Ecabert et al., 2011; Zheng et al., 2008). Furthermore, we would like to emphasize that the current BEAS formulation, based on explicit functions, intrinsically imposes shape limitations on the model, preventing segmentation of the atrial body plus great vessels. For that, a combination of different explicit functions (one function to the body and another to each vessel) would be required. As expected, the errors significantly reduce from the entire mesh situation until the totally cropped version. The biggest differences were found when the appendage's bounding box was considered, which is explained by the variable and complex anatomy found (Tobon-Gomez et al., 2015) (Table 3). Nevertheless, previous segmentation strategies showed similar limitations to capture the appendage region (Ecabert et al., 2011; Tobon-Gomez et al., 2015; Zheng et al., 2008). Moreover, the crop through the valve plane showed a small reduction on the final error result, proving the robustness of the initialization technique, i.e. the use of the ventricular region during BEAS-threshold to compete with the atria and consequently define the valve planes.

An inferior performance was found for the RA body (Table 3 and Fig. 6). Several issues are typically described in RA segmentation (Ecabert et al., 2011; Zheng et al., 2008), namely: 1) difficulties to distinguish between vena cava region and atrial body, 2) noisy blood pool due to the contrast in certain regions, and 3) cumbersome tricuspid valve identification. Nevertheless, the proposed initialization strategy proved to be able to deal with these issues, where the well-defined and well-contrasted left heart works as a barrier for the right heart. The competitive approach intrinsically includes spatial constraints between contours, easing and improving the segmentation of noisy regions as typically found in the RA. As such, while the left heart is allowed to freely evolve due to the high signal-to-noise ratio found in the LA body and the clear edge map obtained, the right heart contour is always controlled by the left heart during the BEAS-threshold. Thus, contours' overlap is prevented, by having the right heart surfaces pushed when initialized inside the left heart region.

Fig. 4 evidenced the importance of this competitive initialization for RA segmentation, with significant differences between the strategy with and without competition. In fact, a competitive strategy with confidence terms was never presented before. Furthermore, our competitive methodology is able to deal with all BEAS shapes (i.e. cylindrical, spherical, among others), being a clear advantage and an added value for this generic framework. We would also like to emphasize that the required minimal thickness parameter $\left(R_{T}\right)$ does not have a high influence in the final segmentation result (Fig. 8a). Since $R_{T}$ is only used as a threshold value to initialize the competition process, and since walls thinner than $R_{T}$ are still allowed in the current implementation, optimal selection of the $R_{T}$ parameter is not required. However, for extreme values, differences are observed due to a high penalization. Regarding the computational time, although the competitive approach is significantly slower than the normal free-evolution BEAS, it is still attractive and notably faster than previously presented multi-structure segmentation techniques with competitive contours (Faisal et al., 2015).

Similar observations were obtained for MRI datasets (Table 4). However, larger errors were obtained when compared with the CT 


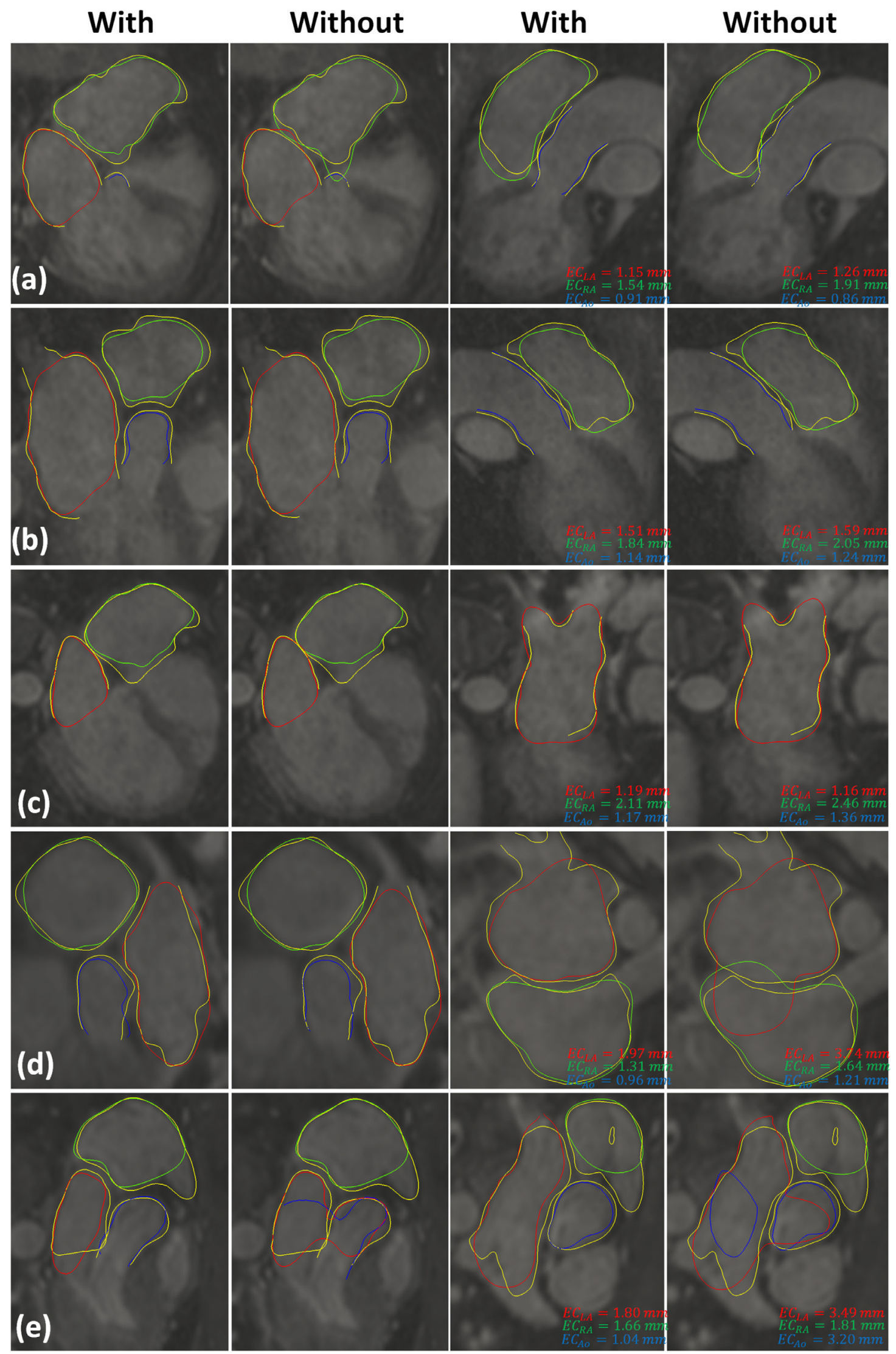

Fig. 13. Segmentation results obtained with and without competitive BEAS technique for the cases corresponding to the 10th (a), 30th (b), 50th (c), 70th (d), and 90th (e) percentiles, according to the average P2S error. Semi-automatic left atrium is represented as red, the right atrium as green and blue is used for the aortic tract. Yellow contours represent manual delineations. The error is presented using a cropped surface (removing bounding boxes and regions below the valve plane). (For interpretation of the references to colour in this figure legend, the reader is referred to the web version of this article.) 
results. A similar trend (i.e. higher errors in MR data) was found for the remaining state-of-the-methods that segment both CT/MR images and even in terms of inter-observer variability. MR segmentation is more challenging than CT due to the larger pixel size (approximately four times larger than in $\mathrm{CT}$ ) and inter-slice spacing and the noisy blood pools found in MR (Fig. 13), as clearly stated in Tobon-Gomez et al. (2015). Furthermore, the performance of the edge map is suboptimal when compared to the CT one due to the multiple transitions found in the atrial bodies, and the holes found in the thin walls. In this sense, and in opposition to the observed in CT, significant differences were found in the entire atrial region between the free-evolution and the competitive BEAS approaches (Fig. 11). In order to increase the accuracy of the atrial segmentation, we believe that a better initialization (anatomic initialization instead of a point in the middle of the atrial body) and even a complete segmentation of the atrial anatomy (body plus vessels) would be required. Regarding the LA benchmark for MR (Fig. 10) (Tobon-Gomez et al., 2015), the proposed methodology proved to be competitive against the best strategies, and similar to the inter-observer variability. It should be noticed that only a few methods were able to accurately segment the LA in both CT and $\mathrm{MR}$, all being computationally demanding strategies, such as atlasbased techniques (Tobon-Gomez et al., 2015; Zuluaga et al., 2013), and requiring reference/atlas cases during the segmentation stage, which constraints the versatility and robustness of the method to the shape variability found in the reference cases. Nevertheless, the proposed methodology is computationally more attractive and no strong assumption (i.e. no training dataset) is required, with the relative position between the different cavities of the atrial region being sufficient to achieve successful results. Similarly to the CT evaluation, we also assessed the influence of the estimated $R_{T}$ parameter in the final MR result (Fig. 8b), and no significant differences were found between the selected parameter (i.e. $2 \mathrm{~mm}$ ) and the neighboring options. This result proved that exhaustive tuning of this parameter is not mandatory, proving the robustness of this strategy. Indeed and although different $R_{T}$ values could be selected for the inter-atrial and aortic wall problems, this result also justifies why we applied the same wall thickness for both scenarios.

Overall, the added-value of the proposed competitive approach was easily observed for the segmentation of atrial region and its heterogeneous thin mid walls (Figs. 5, 12 and 13). Merged contours were always prevented by the proposed approach, which was not obtained by the traditional approach. Moreover, the need for competitive approaches was more pronounced in noisy situations (e.g., inter-atrial septal wall in MRI, Fig. 13). In these situations (i.e. missing walls), total overlap between contours is obtained with the free evolution strategy (Fig. 13e), since no boundaries/transitions are found during the segmentation process. Nevertheless, it should be noted that correct segmentation of the mid-thin walls is not only caused by the competitive formulation, depending on the remaining segmentation terms too. The competitive approach only locally constrains the contour evolution when they are too near, reducing the step size used throughout the contour evolution and allowing a correct refinement of the contours to the real anatomy.

Finally, we would like to mention that the implementation of this technique in a different framework (such as the traditional level sets) is also a viable option, allowing the application of the competitive method for segmentation of more complex shape scenarios (see Appendix A). Moreover, although the proposed method was proven to be accurate for atrial region segmentation in MR and $\mathrm{CT}$, the competitive approach is generic and it also shows potential for a multitude of applications in multiple imaging modalities, such as: segmentation of left ventricular myocardial wall ((Queirós et al., 2014), see multimedia file 1), segmentation of both "true" and "false" lumen in aortic dissection ((Chen et al., 2013), see multimedia file 2), carotid artery bifurcation wall (Arias-Lorza et al., 2016), pelvic cavity (Ma et al., 2012), among others.

\section{Conclusions}

The proposed competitive approach proved to be suitable for atrial region segmentation problems with results similar to other state-of-the-art methods. No merging/overlap of the multiple contours was obtained, which was not possible with the free-evolution version. Moreover and in opposition to the remaining methods described in the literature, the proposed framework showed its clear added-value for the segmentation of mid thin walls. Finally, segmentation of heterogeneous/noisy regions, bad image quality and missed walls cases were significantly improved with the proposed competitive approach.

\section{Conflict of interest}

None.

\section{Acknowledgements}

The authors acknowledge Fundacão para a Ciência e a Tecnologia (FCT), in Portugal, and the European Social Found, European Union, for funding support through the "Programa Operacional Capital Humano" (POCH) in the scope of the $\mathrm{PhD}$ grants SFRH/BD/95438/2013 (P. Morais) and SFRH/BD/93443/2013 (S. Queirós).

Authors gratefully acknowledge the funding of projects NORTE01-0145-FEDER-000013 and NORTE-01-0145-FEDER-000022, co-financed by "Programa Operacional Regional do Norte" (NORTE2020), through "Fundo Europeu de Desenvolvimento Regional" (FEDER).

\section{Appendix A. - Generalization for a standard level-set framework}

In a standard level-set framework as described by Lankton and Tannenbaum (2008), the general expression of its energy is formulated as:

$E=\int_{\Omega} \delta_{\phi}(\boldsymbol{x}) \int_{\Omega} B(\boldsymbol{x}, \boldsymbol{y}) \cdot F(\boldsymbol{y}) d \boldsymbol{y} d \boldsymbol{x}$,

with,

$F(\boldsymbol{y})=f_{\text {in }}(\boldsymbol{y}) \cdot H_{\phi}(\boldsymbol{y})+f_{\text {out }}(\boldsymbol{y}) \cdot\left(1-H_{\phi}(\boldsymbol{y})\right)$,

where, $f_{\text {in }}$ and $f_{\text {out }}$ define the energy criteria for the interior and exterior of the interface $\Gamma . H_{\phi}$ is the Heaviside operator applied to the level-set like function $\phi$. Its expansion for multi-structure segmentation problems is straightforward, as described in Eq. (2). Multiple energies (i.e. one per contour $i$ ) are used ( $n$ energies), which are posteriorly combined during the optimization (Eq. (2)).

The curve evolution is computed as the first derivative of each energy with respect to $\phi_{i}$, as exhaustively described in (Lankton and Tannenbaum, 2008):

$\frac{\partial \phi_{i}}{\partial t}(\boldsymbol{x})=\delta_{\phi_{i}}(\boldsymbol{x}) \int_{\Omega} B(\boldsymbol{x}, \boldsymbol{y}) \cdot \nabla_{\phi_{i}(\boldsymbol{y})} F_{i}(\boldsymbol{y}) d \boldsymbol{y}$,

where $\nabla_{\phi(\boldsymbol{y})} F(\boldsymbol{y})$ is the first derivative of $F_{i}$ with respect to $\phi_{i}$.

Similarly to Eq. (17), the competition strategy between $n$ contours can be embedded in this standard framework through:

$\nabla_{\phi_{i}(\boldsymbol{y})} F_{i}(\boldsymbol{y})=\delta_{\phi_{i}(\boldsymbol{y})} \cdot\left(\bar{g}_{i}^{I M}(\boldsymbol{y})+\sum_{m=1, m \neq i}^{n} \alpha_{i \rightarrow m} . \bar{g}_{i \rightarrow m}^{C P T}(\boldsymbol{y})\right)$, 
with $\bar{g}_{i}^{I M}$ representing the image-based terms (e.g. localized ChanVese, among others) and $\bar{g}_{i \rightarrow m}^{C P T}$ the competition term. $\bar{g}_{i \rightarrow m}^{C P T}$ is defined as presented in Eq. (18).

\section{Supplementary materials}

Supplementary material associated with this article can be found, in the online version, at doi:10.1016/j.media.2017.07.007.

\section{References}

Alessandrini, M., Friboulet, D., Basset, O., D’hooge, J., Bernard, O., 2009. Level-set segmentation of myocardium and epicardium in ultrasound images using localized bhattacharyya distance. In: IEEE International Ultrasonics Symposium, Roma, Italy, pp. 2468-2471.

Almeida, N., Friboulet, D., Sarvari, S.I., Bernard, O., Barbosa, D., Samset, E., D’hooge, J., 2016. Left-atrial segmentation from 3-D ultrasound using B-spline explicit active surfaces with scale uncoupling. IEEE Trans. Ultrason. Ferroelectr. Freq. Control 63, 212-221.

Ammar, M., Mahmoudi, S., Chikh, M.A., Abbou, A., 2013. Toward an automatic left atrium localization based on shape descriptors and prior knowledge. In: International Workshop on Statistical Atlases and Computational Models of the Heart. Springer, pp. 42-48.

Arias-Lorza, A.M., Petersen, J., van Engelen, A., Selwaness, M., van der Lugt, A., Niessen, W.J., de Bruijne, M., 2016. Carotid artery wall segmentation in multispectral MRI by coupled optimal surface graph cuts. IEEE Trans. Med. Imag. 35, 901-911.

Baerentzen, J.A., Aanaes, H., 2005. Signed distance computation using the angle weighted pseudonormal. IEEE Trans. Visual. Comput. Graph. 11, 243-253.

Barbosa, D., Bernard, O., Savu, O., Dietenbeck, T., Heyde, B., Claus, P., Friboulet, D., D'hooge, J., 2010. Coupled B-spline active geometric functions for myocardial segmentation: a localized region-based approach. In: 2010 IEEE International Ultrasonics Symposium, pp. 1648-1651.

Barbosa, D., Dietenbeck, T., Schaerer, J., D’hooge, J., Friboulet, D., Bernard, O., 2012. B-spline explicit active surfaces: an efficient framework for real-time 3-D region-based segmentation. IEEE Trans. Image Process. 21, 241-251.

Beinart, R., Abbara, S., Blum, A., Ferencik, M., Heist, K., Ruskin, J., Mansour, M., 2011. Left atrial wall thickness variability measured by CT scans in patients undergoing pulmonary vein isolation. J. Cardiovasc. Electrophysiol. 22, 1232-1236.

Bourier, F., Reents, T., Ammar-Busch, S., Semmler, V., Telishevska, M., Kottmaier, M., et al., 2016. Transseptal puncture guided by CT-derived 3D-augmented fluoroscopy. J. Cardiovasc. Electrophysiol 27 (3), 369-372.

Brox, T., Weickert, J., 2006. Level set segmentation with multiple regions. IEEE Trans. Image Process. 15, 3213-3218.

Canny, J., 1986. A computational approach to edge detection. IEEE Trans. Pattern Anal. Mach. Intell. 679-698.

Chen, D., Müller-Eschner, M., von Tengg-Kobligk, H., Barber, D., Böckler, D., Hose, R., Ventikos, Y., 2013. A patient-specific study of type-B aortic dissection: evaluation of true-false lumen blood exchange. Biomed. Eng. 12, 1 online.

Chen, T., Babb, J., Kellman, P., Axel, L., Kim, D., 2008. Semiautomated segmentation of myocardial contours for fast strain analysis in cine displacement-encoded MRI. IEEE Trans. Med. Imag. 27, 1084-1094.

Ecabert, O., Peters, J., Schramm, H., Lorenz, C., von Berg, J., Walker, M.J., Vembar, M., Olszewski, M.E., Subramanyan, K., Lavi, G., 2008. Automatic model-based segmentation of the heart in CT images. IEEE Trans. Med. Imag. 27, 1189-1201.

Ecabert, O., Peters, J., Walker, M.J., Ivanc, T., Lorenz, C., von Berg, J., Lessick, J., Vembar, M., Weese, J., 2011. Segmentation of the heart and great vessels in CT images using a model-based adaptation framework. Med. Image Anal. 15, 863-876.

Faisal, A., Ng, S.-C., Goh, S.-L., George, J., Supriyanto, E., Lai, K.W., 2015. Multiple LREK active contours for knee meniscus ultrasound image segmentation. IEEE Trans. Med. Imag. 34, 2162-2171.

Gao, Y., Kikinis, R., Bouix, S., Shenton, M., Tannenbaum, A., 2012. A 3D interactive multi-object segmentation tool using local robust statistics driven active contours. Med. Image Anal. 16, 1216-1227.

Hoit, B.D., 2014. Left atrial size and function: role in prognosis. J. Am. College Cardiol. 63, 493-505.

Iglesias, J.E., Sabuncu, M.R., 2015. Multi-atlas segmentation of biomedical images: a survey. Med. Image Anal. 24, 205-219.

Jimenez-del-Toro, O., Müller, H., Krenn, M., Gruenberg, K., Taha, A.A., Winterstein, M., Eggel, I., Foncubierta-Rodríguez, A., Goksel, O., Jakab, A., 2016. Cloud-based evaluation of anatomical structure segmentation and landmark detection algorithms: VISCERAL anatomy benchmarks. IEEE Trans. Med. Imag. 35, 2459-2475.

Kirișli, H., Schaap, M., Klein, S., Papadopoulou, S., Bonardi, M., Chen, C.-H., Weustink, A., Mollet, N., Vonken, E., van der Geest, R., 2010. Evaluation of a multi-atlas based method for segmentation of cardiac CTA data: a large-scale, multicenter, and multivendor study. Med. Phys. 37, 6279-6291.
Lankton, S., Tannenbaum, A., 2008. Localizing region-based active contours. IEEE Trans. Image. Process. 17, 2029-2039.

Ma, Z., Jorge, R.N.M., Mascarenhas, T., Tavares, J.M.R., 2012. Segmentation of female pelvic cavity in axial T2-weighted MR images towards the 3D reconstruction. Int. J. Numer. Methods Biomed. Eng. 28, 714-726.

Malayeri, A.A., Natori, S., Bahrami, H., Bertoni, A.G., Kronmal, R., Lima, J.A., Bluemke, D.A., 2008. Relation of aortic wall thickness and distensibility to cardiovascular risk factors (from the Multi-Ethnic Study of Atherosclerosis [MESA]). Am. J. Cardiol. 102, 491-496.

Melenovsky, V., Hwang, S.-J., Lin, G., Redfield, M.M., Borlaug, B.A., 2014. Right heart dysfunction in heart failure with preserved ejection fraction. Eur. Heart J. 35, 3452-3462.

Morais, P., Vilaça, J.L., Ector, J., D’hooge, J., Tavares, J.M.R., 2017. Novel solutions applied in transseptal puncture: a systematic review. J. Med. Devices 11 (1) 010801.

Okada, T., Linguraru, M.G., Hori, M., Summers, R.M., Tomiyama, N., Sato, Y., 2015. Abdominal multi-organ segmentation from CT images using conditional shape-location and unsupervised intensity priors. Med. Image Anal. 26, 1-18.

Pedrosa, J., Barbosa, D., Heyde, B., Schnell, F., Rösner, A., Claus, P., D’hooge, J., 2017. Left ventricular myocardial segmentation in 3-D ultrasound recordings: effect of different endocardial and epicardial coupling strategies. IEEE Trans. Ultrason. Ferroelectr. Freq. Control 64 (3), 525-536.

Queirós, S., Barbosa, D., Heyde, B., Morais, P., Vilaça, J.L., Friboulet, D., Bernard, O., D'hooge, J., 2014. Fast automatic myocardial segmentation in 4D cine CMR datasets. Med. Image Anal. 18, 1115-1131.

Queirós, S., Dubois, C., Morais, P., Adriaenssens, T., Fonseca, J.C., Vilaça, J.L., D'hooge, J., 2017. Automatic 3D aortic annulus sizing by computed tomography in the planning of transcatheter aortic valve implantation. J. Cardiovasc. Comput. Tomogr. 11 (1), 25-32.

Queirós, S., Papachristidis, A., Barbosa, D., Theodoropoulos, K.C., Fonseca, J.C., Monaghan, M.J., Vilaça, J.L., D'hooge, J., 2016. Aortic valve tract segmentation from 3D-TEE using shape-based B-spline explicit active surfaces. IEEE Trans. Med Imag. 35, 2015-2025.

Rolf, S., Hindricks, G., Sommer, P., Richter, S., Arya, A., Bollmann, A., Kosiuk, J., Koutalas, E., 2014. Electroanatomical mapping of atrial fibrillation: Review of the current techniques and advances. J. Atrial Fibrill. 7.

Sandoval, Z., Betancur, J., Dillenseger, J.-L., 2013. Multi-atlas-based segmentation of the left atrium and pulmonary veins. In: International Workshop on Statistical Atlases and Computational Models of the Heart. Springer, pp. 24-30.

Squillacote, A.H., Ahrens, J., 2007. The paraview guide. Kitware.

Stender, B., Blanck, O., Wang, B., Schlaefer, A., 2013. Model-based segmentation of the left atrium in CT and MRI scans. In: International Workshop on Statistical Atlases and Computational Models of the Heart. Springer, pp. 31-41.

Tobon-Gomez, C., Geers, A.J., Peters, J., Weese, J., Pinto, K., Karim, R., Ammar, M. Daoudi, A., Margeta, J., Sandoval, Z., 2015. Benchmark for algorithms segmenting the left atrium from 3D CT and MRI datasets. IEEE Trans. Med. Imag. 34, 1460-1473.

Wolf, I., Vetter, M., Wegner, I., Böttger, T., Nolden, M., Schöbinger, M., Hastenteufel, M., Kunert, T., Meinzer, H.-P., 2005. The medical imaging interaction toolkit. Med. Image Anal. 9, 594-604.

Wolz, R., Chu, C., Misawa, K., Fujiwara, M., Mori, K., Rueckert, D., 2013. Automated abdominal multi-organ segmentation with subject-specific atlas generation. IEEE Trans. Med. Imag. 32, 1723-1730.

Xu, Z., Burke, R.P., Lee, C.P., Baucom, R.B., Poulose, B.K., Abramson, R.G., Landman, B.A., 2015. Efficient multi-atlas abdominal segmentation on clinically acquired CT with SIMPLE context learning. Med. Image Anal. 24, 18-27.

Yan, P., Shen, W., Kassim, A.A., Shah, M., 2005. Segmentation of neighboring organs in medical image with model competition. In: International Conference on Medical Image Computing and Computer-Assisted Intervention. Springer, pp. 270-277.

Yang, J., Staib, L.H., Duncan, J.S., 2004. Neighbor-constrained segmentation with level set based 3-D deformable models. IEEE Trans. Med. Imag. 23, 940-948.

Yezzi, A., Tsai, A., Willsky, A., 2002. A fully global approach to image segmentation via coupled curve evolution equations. J. Visu. Commun. Image Represent. 13, 195-216.

Zheng, Y., Barbu, A., Georgescu, B., Scheuering, M., Comaniciu, D., 2008. Four-chamber heart modeling and automatic segmentation for 3-D cardiac CT volumes using marginal space learning and steerable features. IEEE Trans. Med. Imag. 27 (11), 1668-1681.

Zhuang, X., Rhode, K.S., Razavi, R.S., Hawkes, D.J., Ourselin, S., 2010. A registration-based propagation framework for automatic whole heart segmentation of cardiac MRI. IEEE Trans. Med. Imag. 29, 1612-1625.

Zuluaga, M.A., Cardoso, M.J., Modat, M., Ourselin, S., 2013. Multi-atlas propagation whole heart segmentation from MRI and CTA using a local normalised correlation coefficient criterion. In: Functional Imaging and Modeling of the Heart. Springer, pp. 174-181. 